\title{
EHRESMANN CONNECTIONS FOR A FOLIATED MANIFOLD AND CERTAIN KINDS OF RECTANGLES WITHOUT TERMINAL VERTEX
}

\author{
NAOYUKI KOIKE
}

\begin{abstract}
We define the notion of a non-extendable rectangle without terminal vertex for a foliated manifold $(M, \mathfrak{F})$ with a complementary distribution $D$ and classify them into non-singular ones and singular ones. It is easy to show that $D$ is an Ehresmann connection in the sense of R. A. Blumenthal and J. J. Hebda if and only if there is no non-extendable rectangle without terminal vertex. One of our purposes is to investigate the existence of singular non-extendable rectangle without terminal vertex. Another purpose is to obtain a new sufficient condition for the orthogonal complementary distribution of a foliation on a Riemannian manifold to be an Ehresmann connection by investigatıng a property of singular non-extendable rectangles without terminal vertex.
\end{abstract}

\section{Introduction}

Throughout this paper, unless otherwise mentioned, we assume that all objects are smooth (i.e., of class $C^{\infty}$ ) and all manifolds are connected ones without boundary. For a foliated manifold $(M, \mathfrak{F})$ with a complementary distribution $D$, R. A. Blumenthal and J. J. Hebda considered a piecewise smooth map $\delta:[0,1] \times[0,1] \rightarrow M$ such that, for every fixed $s_{0}$, the curve $\delta s_{0}:=\delta\left(\cdot, s_{0}\right)$ is a horizontal curve, and, for every fixed $t_{0}$, the curve $\delta_{t_{0}}:=\delta\left(t_{0}, \cdot\right)$ is a vertical curve, where a horizontal curve is a piecewise smooth map from $[0,1]$ to $M$ whose velocity vector field lies in $D$ and a vertical curve is a piecewise smooth map from $[0,1]$ to a leaf of $\mathfrak{F}$. They called such a piecewise smooth map $\delta$ a rectangle. If, for every vertical curve $\alpha$ and every horizontal curve $\beta$ with $\alpha(0)=\beta(0)$, there is the rectangle $\delta$ with $\delta_{0}=\alpha$ and $\delta_{.0}=\beta$, then they called $D$ an Ehresmann connection for $\mathbb{F}$ (see [2]). They proved the following so-called global stability theorem and decomposition theorem (see [2]):

(i) If $\mathfrak{F}$ admits an Ehresmann connection, then the universal coverings of leaves of $\mathfrak{F}$ are diffeomorphic to one another.

(ii) If $D$ is an integrable Ehresmann connection for $\mathfrak{F}$, then for each $p \in M$, there is a covering map $\pi$ of the product manifold $\hat{L}_{p}^{V} \times \hat{L}_{p}^{H}$ onto $M$ satisfying $\pi_{*}\left(T \hat{L}_{p}^{V}\right)=F$ and $\pi_{*}\left(T \hat{L}_{p}^{H}\right)=D$, where $\hat{L}_{p}^{V}$ (resp. $\left.\hat{L}_{p}^{H}\right)$ is the universal covering of

Received September 24, 1998; revised July 5, 1999. 
a leaf of $\mathfrak{F}$ through $p$ (resp. that of the maximal integral manifold of $D$ through $p$ ), $\pi_{*}$ is the differential of $\pi, T \hat{L}_{p}^{V}$ (resp. $T \hat{L}_{p}^{H}$ ) is the tangent bundle of the foliation $\hat{L}_{p}^{V} \times\{\cdot\}\left(\right.$ resp. $\left.\{\cdot\} \times \hat{L}_{p}^{H}\right)$ on $\hat{L}_{p}^{V} \times \hat{L}_{p}^{H}$ and $F$ is the tangent bundle of $\mathfrak{F}$.

Thus we can apply the study of an Ehresmann connection to those of the global stability of a foliation and the decomposition of a manifold into a product manifold (furthermore, the geometric decomposition of a manifold with a geometric structure). Therefore, it is very interesting to investigate what kind of foliation admits an Ehresmann connection.

In this paper, we consider a piecewise smooth map $\delta:[0,1] \times[0,1] \backslash\{(1,1)\} \rightarrow$ $M$ such that, for every fixed $s_{0} \in[0,1)$, the curve $\delta_{s_{0}}$ is a horizontal curve, for every fixed $t_{0} \in[0,1)$, the curve $\delta_{t_{0}}$ is a vertical curve and $\delta_{1}$ (resp. $\delta_{1}$ ) is a horizontal (resp. vertical) curve without terminal point. We shall call such a piecewise smooth map $\delta$ a rectangle without terminal vertex. If there is not a rectangle $\tilde{\delta}$ satisfying $\left.\tilde{\delta}\right|_{([0,1] \times[0,1] \backslash\{(1,1)\})}=\delta$, then we shall say that $\delta$ is nonextendable. By imitating the proof of Proposition 2.3 of [13], it is shown that $D$ is an Ehresmann connection for $\mathfrak{F}$ if and only if there is no non-extendable rectangle without terminal vertex. Thus the study of a non-extendable rectangle without terminal vertex leads to that of an Ehresmann connection. According to Lemma 3.5 of [11], if $\delta$ is a non-extendable rectangle without terminal vertex, then $\lim _{s \rightarrow 1-0} \delta(1, s)$ does not exist. However, $\lim _{t \rightarrow 1-0} \delta(t, 1)$ is possible to exist. If $\lim _{t \rightarrow 1-0} \delta(t, 1)$ exists (resp. does not exist), then we shall say that $\delta$ is singular (resp. non-singular).

Remark. If $\delta$ is singular, then a continuous curve $c:[0,1] \rightarrow M$ defined by

$$
c(t):= \begin{cases}\delta(t, 1) & (0 \leq t<1) \\ \lim _{t \rightarrow 1-0} \delta(t, 1) & (t=1)\end{cases}
$$

is not of class $C^{1}$ at $t=1$. In fact, it is shown in terms of a foliated coordinate neighbourhood about $c(1)$ that $\delta$ is extendable if $c$ is of class $C^{1}$ at $t=1$.

If $\operatorname{codim} \mathfrak{F}=1$, that is, $\operatorname{dim} D=1$, then $D$ is integrable and hence all nonextendable rectangles without terminal vertex are non-singular. It is very important to investigate the existence of a singular non-extendable rectangle without terminal vertex in case of codim $\mathfrak{F} \geq 2$. In this paper, we shall prove the following result related to its existence.

THEOREM 1. For every $r \geq 3$ and every $n \geq r+1$, there is a triple $(M, \mathfrak{F}, D)$ of an n-dimensional manifold $M$, a foliation $\mathfrak{F}$ of codimension $r$ on $M$ and $a$ complementary distribution $D$ to $\mathfrak{F}$ which admits a singular non-extendable rectangle without terminal vertex.

It is natural to ask what kind of foliation admits an Ehresmann connection on a manifold with a geometric structure. On a Riemannian manifold, such a study has been already done by some geometers as follows. Let $F^{\perp}$ be the 
orthogonal complementary distribution of a foliation $\mathfrak{F}$ on a Riemannian manifold $(M, g)$. It is known that $F^{\perp}$ is an Ehresmann connection if one of the following conditions holds (see [2], [4], [8], [15]):

(I) $(M, g)$ is complete and $g$ is bundle-like for $\mathfrak{F}$,

(II) the induced Riemannian metrics on leaves of $\mathfrak{F}$ are complete and $\mathfrak{F}$ is totally geodesic,

(III) $\operatorname{dim} \mathfrak{F} \geq 3$, the induced conformal structures on leaves of $\mathfrak{F}$ are complete and $\mathfrak{F}$ is totally umbilic.

In [7], for each vertical curve $\alpha$, we defined a function $G_{\alpha}^{\perp}$ on the $\operatorname{set} \operatorname{Rec}(\alpha, \cdot)$ of all rectangles $\delta$ such that $\delta_{0}=\alpha$ and $\delta_{.0}$ is a regular curve by $G_{\alpha}^{\perp}(\delta):=l\left(\delta_{1}\right) /$ $l(\delta .0)$ for $\delta \in \operatorname{Rec}(\alpha, \cdot)$, where $l(\cdot)$ is the length of a curve * with respect to $g$. Also, for each horizontal curve $\beta$, we defined a function $G_{\beta}^{T}$ on the $\operatorname{set} \operatorname{Rec}(\cdot, \beta)$ of all rectangles $\delta$ such that $\delta_{._{0}}=\beta$ and $\delta_{0}$. is a regular curve by $G_{\beta}^{T}(\delta):=$ $l\left(\delta_{1}\right) / l\left(\delta_{0}.\right)$ for $\delta \in \operatorname{Rec}(\cdot, \beta)$. In the paper, we proved that $F^{\perp}$ is an Ehresmann connection if one of the following conditions holds:

(IV) $(M, g)$ is complete and $\sup _{s \in[0,1)} \sup G_{\left.\alpha\right|_{[0, s]} ^{\perp}}^{\perp}<\infty$ for every vertical curve $\alpha:[0,1) \rightarrow M$ without terminal point,

$(\mathrm{V})$ the induced Riemannian metrics on leaves of $\mathfrak{F}$ are complete and $\sup G_{\beta}^{T}<\infty$ for every horizontal curve $\beta$.

Here we note that $G_{\alpha}^{\perp} \equiv 1$ holds for every vertical curve $\alpha$ if $g$ is bundle-like for $\mathfrak{F}$ and that $G_{\beta}^{T} \equiv 1$ holds for every horizontal curve $\beta$ if $\mathfrak{F}$ is totally geodesic. Thus these results are generalizations of I and II above. In this paper, we shall furthermore improve one of these results as follows.

THEOREM 2. If $(M, g)$ is complete and $\sup G_{\alpha}^{\perp}<\infty$ for every vertical curve $\alpha$, then $F^{\perp}$ is an Ehresmann connection.

This theorem will be proved by investigating a property of singular nonextendable rectangles without terminal vertex. We shall also give examples showing that this improvement is essential (see §3). Furthermore, we shall give examples showing the topological gap between foliations admitting a Riemannian metric such that $\sup G_{\alpha}^{\perp}<\infty$ for every vertical curve $\alpha$ and foliations admitting a bundle-like metric (see $\$ 3$ ). The following corollary is directly deduced from Theorem 2 and the sufficient condition $(V)$ for $F^{\perp}$ to be an Ehresmann connection.

COROLlaRY. Let $\mathfrak{F}$ be a foliation on a Riemannian manifold $(M, g)$ satisfying the above condition $(V)$ or the assumption of Theorem 2. Then the following statements (i) and (ii) hold:

(i) The universal coverings of leaves of $\mathfrak{F}$ are diffeomorphic to one another.

(ii) If $\operatorname{codim} \mathfrak{F}=1$, then the universal covering of $M$ is diffeomorphic to $\hat{L}_{p}^{V} \times$ $\boldsymbol{R}$, where $p$ is an arbitrary point of $M$ and $\hat{L}_{p}^{V}$ is the universal covering of the leaf of F through $p$.

In $\S 1$ and $\S 2$, we prove Theorems 1 and 2 , respectively. In $\S 3$, we give examples of a non-extendable rectangle without terminal vertex and those of a 
foliated Riemannian manifold which satisfies the assumption of Theorem 2 but does not satisfy the condition (IV). Furthermore, we give examples of a foliated manifold which admits a Riemannian metric satisfying the assumption of Theorem 2 but does not admit a bundle-like metric.

\section{Proof of Theorem 1}

In this section, we shall prove Theorem 1 by constructing a triple $(M, \mathfrak{F}, D)$ admitting a singular non-extendable rectangle without terminal vertex. First we shall present a plan of construction of such a triple $(M, \mathfrak{F}, D)$.

Plan of Construction. Let $\left(x_{1}, \ldots, x_{n}\right)$ be the natural coordinate of an $n$ dimensional affine space $\boldsymbol{R}^{n}$ and $\mathfrak{F}$ a foliation on $\boldsymbol{R}^{n}$ whose leaves are fibres of the projection $\pi: \boldsymbol{R}^{n} \rightarrow \boldsymbol{R}^{r}$ defined by $\pi\left(x_{1}, \ldots, x_{n}\right)=\left(x_{1}, \ldots, x_{r}\right)(r \geq 2, n \geq r+1)$.

(Step I) First we construct a complementary $\left(C^{\infty}\right.$-)distribution $D_{1}$ to $\mathfrak{F}$, a $C^{\infty}$-curve $\beta=\left(\beta_{1}, \ldots, \beta_{r}\right):[0,1) \rightarrow \boldsymbol{R}^{r}$ without terminal point and a $C^{\infty}$-curve $\alpha:[0,1] \rightarrow \pi^{-1}(\beta(0))$ satisfying the following conditions:

(i) $\lim _{t \rightarrow 1-0} \beta(t)$ exists and a continuous curve $\bar{\beta}:[0,1] \rightarrow \boldsymbol{R}^{r}$ defined by

$$
\bar{\beta}(t):= \begin{cases}\beta(t) & (0 \leq t<1) \\ \lim _{t \rightarrow 1-0} \beta(t) & (t=1)\end{cases}
$$

is not of class $C^{1}$ at $t=1$.

(ii) For every $s \in[0,1]$, there is the $D_{1}$-lift $\tilde{\beta}_{s}:[0,1) \rightarrow \boldsymbol{R}^{n}$ of $\beta$ starting from $\alpha(s)$.

(iii) For every $s \in[0,1), \lim _{t \rightarrow 1-0} \sum_{l=r+1}^{n} x_{i}\left(\tilde{\beta}_{s}(t)\right)^{2}=\infty$.

(iv) $\lim _{t \rightarrow 1-0} \tilde{\beta}_{1}(t)$ exists.

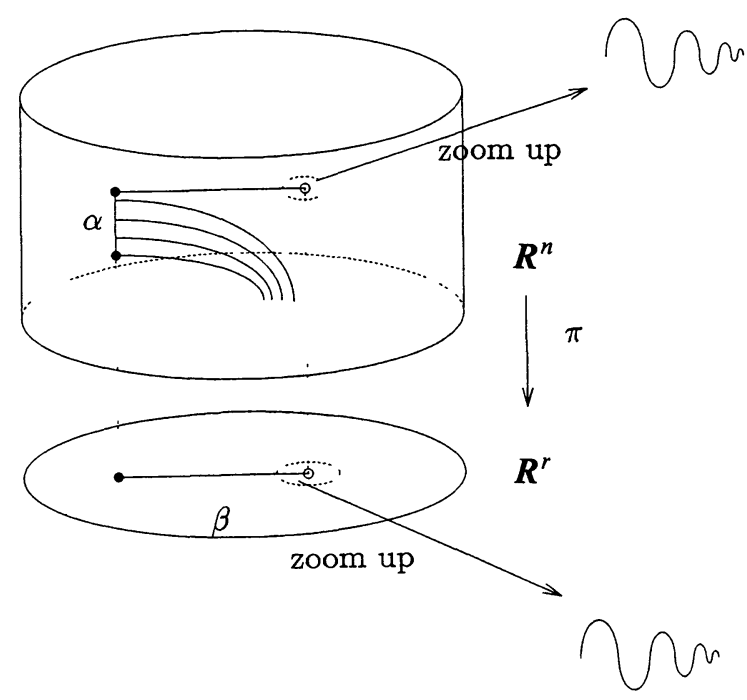

Figure 1.1. 
(Step II) Next, we construct a homeomorphism $\phi$ of $\boldsymbol{R}^{n}$ which admits closed sets $S_{1}$ and $S_{2}$ of $\boldsymbol{R}^{n}$ satisfying the following conditions:

(v) $\left.\phi\right|_{\boldsymbol{R}^{n} \backslash\left(S_{1} \cup S_{2}\right)}$ is a $C^{\infty}$-diffeomorphism.

(vi) $\left(S_{1} \cup S_{2}\right) \cap\left(\pi^{-1}(\beta([0,1))) \cup\left\{\lim _{t \rightarrow 1-0} \tilde{\beta}_{1}(t)\right\}\right)=\emptyset$ and $S_{2} \cap$ $\pi^{-1}\left(\lim _{t \rightarrow 1-0} \beta(t)\right) \neq \emptyset$.

(vii) Let $\gamma=\left(\gamma_{1}, \ldots, \gamma_{n-r}\right):[0,1] \rightarrow \boldsymbol{R}^{n-r}$ be an arbitrary $C^{\infty}$-curve in $\boldsymbol{R}^{n-r}$ with $\gamma(1) \in \pi^{\prime}\left(S_{2} \cap \pi^{-1}\left(\lim _{t \rightarrow 1-0} \beta(t)\right)\right)$ and $\beta_{\gamma}$ a continuous curve in $\boldsymbol{R}^{n}$ defined by

$$
\beta_{\gamma}(t):= \begin{cases}\left(\beta_{1}(t), \ldots, \beta_{r}(t), \gamma_{1}(t), \ldots, \gamma_{n-r}(t)\right) & (0 \leq t<1) \\ \left(\lim _{t \rightarrow 1-0} \beta_{1}(t), \ldots, \lim _{t \rightarrow 1-0} \beta_{r}(t), \gamma_{1}(1), \ldots, \gamma_{n-r}(1)\right) & (t=1),\end{cases}
$$

where $\pi^{\prime}$ is the projection of $\boldsymbol{R}^{n}$ onto $\boldsymbol{R}^{n-r}$ defined by $\pi^{\prime}\left(x_{1}, \ldots, x_{n}\right)=\left(x_{r+1}, \ldots\right.$, $\left.x_{n}\right)$. Then $\phi \circ \beta_{\gamma}$ is of class $C^{\infty}$ over $[0,1]$.

(viii) Give $\boldsymbol{R}^{n} \backslash S_{1}$ a $C^{\infty}$-structure $\left\{\left(\boldsymbol{R}^{n} \backslash S_{1},\left.\phi\right|_{\boldsymbol{R}^{n} \backslash S_{1}}\right)\right\}$. Denote this $C^{\infty}$ manifold by $M$. Then $\mathfrak{F}$ becomes a $\left(C^{\infty}-\right)$ foliation on $M$.

(Step III) Furthermore, we construct a complementary $\left(C^{\infty}\right.$-)distribution $D$ to $\mathfrak{F}$ on $M$ satisfying the following conditions:

(ix) $D=D_{1}$ on a neighbourhood of $\tilde{\beta}_{1}([0,1)) \cup\left\{\lim _{t \rightarrow 1-0} \tilde{\beta}_{1}(t)\right\}$. $\alpha(s)$.

(x) For every $s \in[0,1)$, there is the $D$-lift $\beta_{s}^{L}:[0,1) \rightarrow M$ of $\beta$ starting from

(xi) For every $s \in[0,1), \lim _{t \rightarrow 1-0} \beta_{s}^{L}(t)$ exists and $\lim _{t \rightarrow 1-0} \pi^{\prime}\left(\beta_{s}^{L}(t)\right)$ belongs to $\pi^{\prime}\left(S_{2} \cap \pi^{-1}\left(\lim _{t \rightarrow 1-0} \beta(t)\right)\right)$.

(xii) Let $\widehat{\pi}^{\prime} \circ \beta_{s}^{L}:[0,1] \rightarrow \boldsymbol{R}^{n-r}(s \in[0,1))$ be a continuous curve in $\boldsymbol{R}^{n-r}$ defined by

$$
\left({\widehat{\pi^{\prime} \circ \beta_{s}}}^{L}\right)(t):= \begin{cases}\left(\pi^{\prime} \circ \beta_{s}^{L}\right)(t) & (0 \leq t<1) \\ \lim _{t \rightarrow 1-0}\left(\pi^{\prime} \circ \beta_{s}^{L}\right)(t) & (t=1) .\end{cases}
$$

Then $\widehat{\pi}^{\prime} \circ \beta_{s}^{L}$ becomes a $C^{\infty}$-curve for every $s \in[0,1)$.

Then we define a map $\delta:([0,1] \times[0,1] \backslash\{(1,1)\}) \rightarrow M$ by

$$
\delta(t, s):= \begin{cases}\beta_{s}^{L}(t) & (0 \leq t<1,0 \leq s<1) \\ \lim _{t \rightarrow 1-0} \beta_{s}^{L}(t) & (t=1,0 \leq s<1) \\ \tilde{\beta}_{1}(t) & (0 \leq t<1, s=1) .\end{cases}
$$

It follows from the definition of $\delta$ that $\delta_{s}(s \in[0,1))$ are given by

$$
\delta \cdot s(t)= \begin{cases}\beta_{s}^{L}(t) & (0 \leq t<1) \\ \lim _{t \rightarrow 1-0} \beta_{s}^{L}(t) & (t=1)\end{cases}
$$

which is a $C^{\infty}$-curve in $M$ by the conditions (vii), (xi) and (xii). Hence 


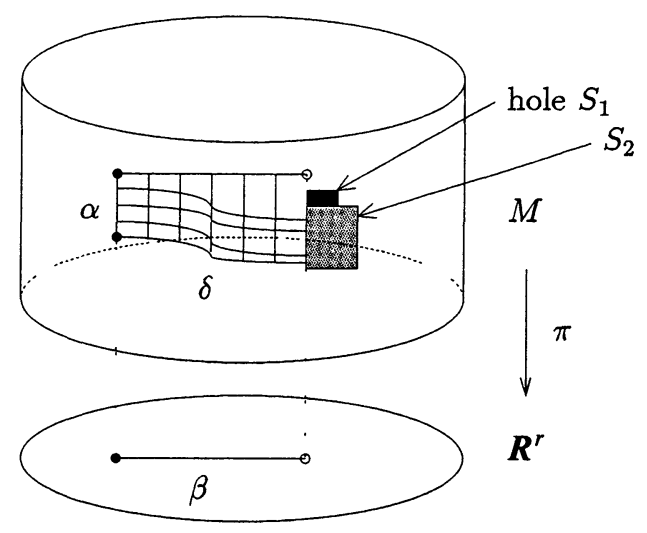

Figure 1.2.

$\delta_{s}(s \in[0,1))$ are horizontal curves (with respect to $\left.D\right)$. Also, it follows from the conditions (v), (vi) and (ix) that $\tilde{\beta}_{1}(=\delta .1)$ is a horizontal curve (with respect to $D$ ) without terminal point. These facts imply that $\delta$ is a rectangle without terminal vertex on $(M, \mathfrak{F}, D)$. By the condition (iv), $\lim _{t \rightarrow 1-0} \delta(t, 1)$ exists. Moreover, by the conditions (i), (v) and (vi), a continuous curve $\bar{\delta}_{1}:[0,1] \rightarrow M$ defined by

$$
\bar{\delta}_{1}(t)= \begin{cases}\delta_{1}(t) & (0 \leq t<1) \\ \lim _{t \rightarrow 1-0} \delta(t, 1) & (t=1)\end{cases}
$$

is not of class $C^{1}$ at $t=1$. This fact implies that $\delta$ is non-extendable and singular. Thus this triple $(M, \mathfrak{F}, D)$ admits a singular non-extendable rectangle $\delta$ without terminal vertex.

Proof of Theorem 1. Following to the above plan of construction, we shall concretely construct a triple $(M, \mathfrak{F}, D)$ which admits a singular non-extendable rectangle without terminal vertex in case of $r \geq 3$. Let $\mathfrak{F},\left(x_{1}, \ldots, x_{n}\right), \pi$ and $\pi^{\prime}$ be as above. First we define a complementary $\left(C^{\infty}\right.$-)distribution $D_{1}$ to $\mathfrak{F}$ on $\boldsymbol{R}^{n}$, a $C^{\infty}$-curve $\beta=\left(\beta_{1}, \ldots, \beta_{r}\right):[0,1) \rightarrow \boldsymbol{R}^{r}$ without terminal point and a $C^{\infty}$-curve $\alpha:[0,1] \rightarrow \pi^{-1}(\beta(0))$ by

$$
\begin{gathered}
D_{1}:=\operatorname{Span}\left\{\frac{\partial}{\partial x_{1}}, \frac{\partial}{\partial x_{2}}+x_{3} x_{n} \frac{\partial}{\partial x_{n}}, \frac{\partial}{\partial x_{3}}-x_{2} x_{n} \frac{\partial}{\partial x_{n}}, \frac{\partial}{\partial x_{4}}, \ldots, \frac{\partial}{\partial x_{r}}\right\}, \\
\beta(t):=\left(t-1,(t-1) \sin \frac{1}{(t-1)^{2}},(t-1) \cos \frac{1}{(t-1)^{2}}, 0, \ldots, 0\right) \quad(0 \leq t<1), \\
\alpha(s):=(-1,-\sin 1,-\cos 1,0, \ldots, 0, s-1),
\end{gathered}
$$

respectively. Clearly $\beta$ satisfies the condition (i). The $D_{1}$-lift $\tilde{\beta}_{s}$ of $\beta$ starting 
from $\alpha(s)$ is given by

$$
\tilde{\beta}_{s}(t)=\left(t-1,(t-1) \sin \frac{1}{(t-1)^{2}},(t-1) \cos \frac{1}{(t-1)^{2}}, 0, \ldots, 0, \frac{s-1}{(1-t)^{2}}\right),
$$

where $s \in[0,1]$. Hence $\tilde{\beta}_{s}(s \in[0,1))$ is defined over $[0,1)$. Furthermore, we have

$$
\begin{gathered}
\lim _{t \rightarrow 1-0} \sum_{l=r+1}^{n} x_{i}\left(\tilde{\beta}_{s}(t)\right)^{2}=\lim _{t \rightarrow 1-0} \frac{(s-1)^{2}}{(1-t)^{4}}=\infty \quad(s \in[0,1)), \\
\lim _{t \rightarrow 1-0} \tilde{\beta}_{1}(t)=(0, \ldots, 0) .
\end{gathered}
$$

Thus the conditions (ii)-(iv) hold.

Next we define a homeomorphism $\phi=\left(y_{1}, \ldots, y_{n}\right)$ of $\boldsymbol{R}^{n}$ by

$$
\phi\left(x_{1}, \ldots, x_{n}\right):=\left(x_{1}, \mu\left(2 x_{1}+x_{2}, \lambda\left(x_{n}\right)\right), \mu\left(2 x_{1}+x_{3}, \lambda\left(x_{n}\right)\right), x_{4}, \ldots, x_{n}\right),
$$

where $\mu$ is a $C^{\infty}$-function over $\boldsymbol{R}^{2}$ defined by

$$
\mu(z, w):= \begin{cases}z \cdot e^{-\left(w^{2} /|z|\right)} & (z \neq 0) \\ 0 & (z=0)\end{cases}
$$

and $\lambda$ is a $C^{\infty}$-function over $\boldsymbol{R}$ with $\lambda^{-1}(0)=[-(1 / 2), \infty), \lambda^{-1}(1)=(-\infty,-1]$ and $0 \leq \lambda \leq 1$. Now we shall show that $\phi$ admits closed sets $S_{i}(i=1,2)$ of $\boldsymbol{R}^{n}$ satisfying the above conditions (v)-(viii). Define closed sets $S_{i}(i=1,2)$ of $\boldsymbol{R}^{n}$ by

$$
S_{1}:=\left\{\left(x_{1}, \ldots, x_{n}\right) \mid\left(2 x_{1}+x_{2}\right)\left(2 x_{1}+x_{3}\right)=0 \text { and }-1 \leq x_{n} \leq-\frac{1}{2}\right\}
$$

and

$$
S_{2}:=\left\{\left(x_{1}, \ldots, x_{n}\right) \mid\left(2 x_{1}+x_{2}\right)\left(2 x_{1}+x_{3}\right)=0 \text { and } x_{n} \leq-1\right\}
$$

respectively. Clearly $S_{1}$ and $S_{2}$ satisfy the conditions (v) and (vi). Take an arbitrary $C^{\infty}$-curve $\gamma=\left(\gamma_{1}, \ldots, \gamma_{n-r}\right):[0,1] \rightarrow \boldsymbol{R}^{n-r}$ with $\gamma(1) \in \pi^{\prime}\left(S_{2} \cap\right.$ $\left.\pi^{-1}\left(\lim _{t \rightarrow 1-0} \beta(t)\right)\right)$. Let $\beta_{\gamma}$ be a continuous curve defined as in (vii). We must show that $\phi \circ \beta_{\gamma}$ is of class $C^{\infty}$ over $[0,1]$. Since $\gamma(1) \in \pi^{\prime}\left(S_{2} \cap\right.$ $\left.\pi^{-1}\left(\lim _{t \rightarrow 1-0} \beta(t)\right)\right)$ and hence $\gamma_{n-r}(1)<-1$, we see that $\gamma_{n-r}<-1$ holds over $(1-\varepsilon, 1]$ for a sufficiently small $\varepsilon>0$. Hence we have $\lambda \circ \gamma_{n-r}=1$ over $(1-\varepsilon, 1]$, that is,

$$
\left(\phi \circ \beta_{\gamma}\right)(t)= \begin{cases}\left(t-1,(t-1)\left(2+\sin \frac{1}{(t-1)^{2}}\right) e^{-1 /\left|(t-1)\left(2+\sin \left(1 /(t-1)^{2}\right)\right)\right|}\right. & \\ (t-1)\left(2+\cos \frac{1}{(t-1)^{2}}\right) e^{-1 /\left|(t-1)\left(2+\cos \left(1 /(t-1)^{2}\right)\right)\right|}, & \\ \left.0, \ldots, 0, \gamma_{1}(t), \ldots, \gamma_{n-r}(t)\right) & (1-\varepsilon<t<1) \\ \left(0, \ldots, 0, \gamma_{1}(1), \ldots, \gamma_{n-r}(1)\right) & (t=1) .\end{cases}
$$


This implies that $\phi \circ \beta_{\gamma}$ is of class $C^{\infty}$ over $(1-\varepsilon, 1]$ and hence so is it over $[0,1]$. Thus $S_{1}$ and $S_{2}$ satisfy the condition (vii). Since $\left.\phi\right|_{\boldsymbol{R}^{n} \backslash\left(S_{1} \cup S_{2}\right)}$ is a $C^{\infty}$-diffeomorphism, $\mathfrak{F}$ becomes a foliation on $M \backslash S_{2}$. Let $\mathfrak{F}_{1}$ be a foliation on $M$ whose leaves are the fibres of the projection $\pi_{1}: M \rightarrow \boldsymbol{R}^{r}$ defined by $\pi_{1}\left(x_{1}, \ldots, x_{n}\right)=$ $\left(y_{1}\left(x_{1}, \ldots, x_{n}\right), \ldots, y_{r}\left(x_{1}, \ldots, x_{n}\right)\right)\left(\left(x_{1}, \ldots, x_{n}\right) \in M\right)$. Set $W:=\left\{\left(x_{1}, \ldots, x_{n}\right) \in M\right.$ $\left.\mid x_{n}<-1\right\}$. On $W, \phi\left(x_{1}, \ldots, x_{n}\right)=\left(x_{1}, \mu\left(2 x_{1}+x_{2}, 1\right), \mu\left(2 x_{1}+x_{3}, 1\right), x_{4}, \ldots, x_{n}\right)$ holds. This implies that $\mathfrak{F}=\mathfrak{F}_{1}$ on $W$. Therefore, $\mathfrak{F}$ becomes a foliation on $\left(M \backslash S_{2}\right) \cup W=M$. Thus $S_{1}$ and $S_{2}$ satisfy the condition (viii).

Next we shall construct a complementary distribution $D$ to $\mathfrak{F}$ on $M$ satisfying the conditions (ix)-(xii). Let $\left\{U_{1}, U_{2}\right\}$ be an open covering of $M$ defined by

$$
\begin{aligned}
U_{1}:= & \left\{\left(x_{1}, \ldots, x_{n}\right) \in M \mid x_{n} \geq-1\right. \text { or } \\
& \left.\left(-2<x_{n}<-1 \text { and }\left(2 x_{1}+x_{2}\right)\left(2 x_{1}+x_{3}\right) \neq 0\right)\right\}, \\
U_{2}:= & \left\{\left(x_{1}, \ldots, x_{n}\right) \in M \mid x_{n}<-1\right\}
\end{aligned}
$$

and $\left\{\eta_{1}, \eta_{2}\right\}$ a partition of unity subordinating to $\left\{U_{1}, U_{2}\right\}$. Set

$$
\begin{aligned}
X_{1}:= & \frac{\partial}{\partial y_{1}}+\eta_{1}\left(\frac{\partial y_{2}}{\partial x_{1}} \frac{\partial}{\partial y_{2}}+\frac{\partial y_{3}}{\partial x_{1}} \frac{\partial}{\partial y_{3}}\right), \\
X_{2}:= & \left(\eta_{1} \frac{\partial y_{2}}{\partial x_{2}}+\eta_{2}\right) \frac{\partial}{\partial y_{2}}+\eta_{1} x_{3} x_{n}\left(\frac{\partial y_{2}}{\partial x_{n}} \frac{\partial}{\partial y_{2}}+\frac{\partial y_{3}}{\partial x_{n}} \frac{\partial}{\partial y_{3}}+\frac{\partial}{\partial y_{n}}\right) \\
& -\frac{\eta_{1} \eta_{2} x_{3} x_{n}}{\eta_{1} \frac{\partial y_{3}}{\partial x_{3}}+\eta_{2}} \frac{\partial}{\partial y_{n}}, \\
X_{3}:= & \left(\eta_{1} \frac{\partial y_{3}}{\partial x_{3}}+\eta_{2}\right) \frac{\partial}{\partial y_{3}}-\eta_{1} x_{2} x_{n}\left(\frac{\partial y_{2}}{\partial x_{n}} \frac{\partial}{\partial y_{2}}+\frac{\partial y_{3}}{\partial x_{n}} \frac{\partial}{\partial y_{3}}+\frac{\partial}{\partial y_{n}}\right) \\
& +\frac{\eta_{1} \eta_{2} x_{2} x_{n}}{\eta_{1} \frac{\partial y_{2}}{\partial x_{2}}+\eta_{2}} \frac{\partial}{\partial y_{n}} .
\end{aligned}
$$

Since $x_{l}, \partial y_{i} / \partial x_{1}, \partial y_{i} / \partial x_{l}, \partial y_{l} / \partial x_{n}(i=2,3)$ are $C^{\infty}$-functions on $M \backslash S_{2}$ and $\eta_{1}=0$ on a neighbourhood of $S_{2}$, we see that $\eta_{1} x_{l}, \eta_{1}\left(\partial y_{i} / \partial x_{1}\right), \eta_{1}\left(\partial y_{i} / \partial x_{l}\right)$, $\eta_{1}\left(\partial y_{i} / \partial x_{n}\right)(i=2,3)$ are $C^{\infty}$-functions on $M$. Also, it is clear that $x_{n}$ is a $C^{\infty}$ function on $M$. Furthermore, for $i=2,3$, we have

$$
\frac{\partial y_{i}}{\partial x_{l}}= \begin{cases}\left(1+\frac{\lambda\left(x_{n}\right)}{\left|2 x_{1}+x_{i}\right|}\right) \cdot e^{-\lambda\left(x_{n}\right) /\left|2 x_{1}+x_{l}\right|} & \left(2 x_{1}+x_{l} \neq 0\right) \\ 1 & \left(2 x_{1}+x_{l}=0, x_{n} \geq-\frac{1}{2}\right) \\ 0 & \left(2 x_{1}+x_{l}=0, x_{n}<-\frac{1}{2}\right)\end{cases}
$$


and hence $\eta_{1}\left(\partial y_{i} / \partial x_{l}\right)+\eta_{2}>0(i=2,3)$ hold on $M$. Thus $X_{l}(i=1,2,3)$ are $C^{\infty}$-vector fields on $M$ and furthermore $\left(X_{1}, X_{2}, X_{3}, \partial / \partial y_{4}, \ldots, \partial / \partial y_{n}\right)$ is a frame field on $M$. Define a $\left(C^{\infty}\right.$-)distribution $D$ on $M$ by $D:=\operatorname{Span}\left\{X_{1}, X_{2}, X_{3}\right.$, $\left.\partial / \partial y_{4}, \ldots, \partial / \partial y_{r}\right\}$. Since the tangent bundle of $\mathfrak{F}$ is given by $\operatorname{Span}\left\{\partial / \partial y_{r+1}, \ldots\right.$, $\left.\partial / \partial y_{n}\right\}$, we see that $D$ is a complementary distribution to $\mathfrak{F}$. First we shall show that $D$ satisfies the condition (ix). Since $\left.\phi\right|_{\boldsymbol{R}^{n} \backslash\left(S_{1} \cup S_{2}\right)}$ is a $C^{\infty}$-diffeomorphism, $\partial / \partial x_{l}=\sum_{k=1}^{n}\left(\partial y_{k} / \partial x_{l}\right)\left(\partial / \partial y_{k}\right) \quad(1 \leq i \leq n)$ hold on $M \backslash S_{2}$. In more detail, we can obtain

$$
\begin{aligned}
& \frac{\partial}{\partial x_{1}}=\frac{\partial}{\partial y_{1}}+\frac{\partial y_{2}}{\partial x_{1}} \frac{\partial}{\partial y_{2}}+\frac{\partial y_{3}}{\partial x_{1}} \frac{\partial}{\partial y_{3}} \\
& \frac{\partial}{\partial x_{2}}=\frac{\partial y_{2}}{\partial x_{2}} \frac{\partial}{\partial y_{2}}, \quad \frac{\partial}{\partial x_{3}}=\frac{\partial y_{3}}{\partial x_{3}} \frac{\partial}{\partial y_{3}}, \quad \frac{\partial}{\partial x_{i}}=\frac{\partial}{\partial y_{i}} \quad(4 \leq i \leq r)
\end{aligned}
$$

and

$$
\frac{\partial}{\partial x_{j}}=\frac{\partial}{\partial y_{j}} \quad(r+1 \leq j \leq n-1), \quad \frac{\partial}{\partial x_{n}}=\frac{\partial y_{2}}{\partial x_{n}} \frac{\partial}{\partial y_{2}}+\frac{\partial y_{3}}{\partial x_{n}} \frac{\partial}{\partial y_{3}}+\frac{\partial}{\partial y_{n}}
$$

on $M \backslash S_{2}$. Since $\eta_{1}=1$ and $\eta_{2}=0$ on $M \backslash U_{2}$, we have $X_{1}=\partial / \partial x_{1}, X_{2}=\partial / \partial x_{2}+$ $x_{3} x_{n}\left(\partial / \partial x_{n}\right)$ and $X_{3}=\partial / \partial x_{3}-x_{2} x_{n}\left(\partial / \partial x_{n}\right)$ on $M \backslash U_{2}$. Hence $D=D_{1}$ holds on $M \backslash U_{2}$. Since $M \backslash U_{2}$ is a neighbourhood of $\tilde{\beta}_{1}([0,1)) \cup\left\{\lim _{t \rightarrow 1-0} \tilde{\beta}(t)\right\}, D$ satisfies the condition (ix). Next we shall show that $D$ satisfies the conditions $(\mathrm{x})-(\mathrm{xii})$. Let $\beta_{s}^{L}$ (resp. $\tilde{\beta}_{s}$ ) be the $D$-lift (resp. the $D_{1}$-lift) of $\beta$ starting from $\alpha(s)$. Fix $s \in[0,1)$. Set $t_{0}:=\sup \left\{t \mid \beta_{s}^{L}\right.$ is defined over $\left.[0, t]\right\}$. Set $t_{1}:=\sup \left\{t \in\left[0, t_{0}\right) \mid\right.$ $\left.\beta_{s}^{L}([0, t]) \subset M \backslash U_{2}\right\}$. Since $D=D_{1}$ on $M \backslash U_{2}$, we have $\beta_{s}^{L}=\tilde{\beta}_{s}$ on $\left[0, t_{1}\right)$. From $\lim _{t \rightarrow 1-0} x_{n}\left(\tilde{\beta}_{s}(t)\right)=\lim _{t \rightarrow 1-0}(s-1) /(1-t)=-\infty$, we have $t_{1}<t_{0}$. We can express $\beta_{s}^{L}$ as

$$
\begin{aligned}
\beta_{s}^{L}(t)= & \left(t-1,(t-1) \sin \frac{1}{(t-1)^{2}},(t-1) \cos \frac{1}{(t-1)^{2}},\right. \\
& \left.0, \ldots, 0, x_{r+1}\left(\beta_{s}^{L}(t)\right), \ldots, x_{n}\left(\beta_{s}^{L}(t)\right)\right) \quad\left(t \in\left[0, t_{0}\right)\right) .
\end{aligned}
$$

Then we have

$$
\begin{aligned}
\dot{\beta}_{s}^{L}(t)= & \frac{\partial}{\partial x_{1}}+\left(\sin \frac{1}{(t-1)^{2}}-\frac{2}{(t-1)^{2}} \cos \frac{1}{(t-1)^{2}}\right) \frac{\partial}{\partial x_{2}} \\
& +\left(\cos \frac{1}{(t-1)^{2}}+\frac{2}{(t-1)^{2}} \sin \frac{1}{(t-1)^{2}}\right) \frac{\partial}{\partial x_{3}} \\
& +\sum_{t=r+1}^{n} \frac{d\left(x_{l} \circ \beta_{s}^{L}\right)}{d t} \frac{\partial}{\partial x_{l}} \quad\left(t \in\left[0, t_{0}\right)\right) .
\end{aligned}
$$

Set $I:=\left\{t \in\left[0, t_{0}\right) \mid \beta_{s}^{L}(t) \in \bar{U}_{2}\right\}$. It follows from $y_{i}=\mu\left(2 x_{1}+x_{i}, \lambda\left(x_{n}\right)\right)(i=2,3)$ 
and $\left.\left(\lambda \circ x_{n}\right)\right|_{\bar{U}_{2}}=1$ that

$$
\left(\frac{\partial y_{2}}{\partial x_{n}} \circ \beta_{s}^{L}\right)(t)=\left(\frac{\partial y_{3}}{\partial x_{n}} \circ \beta_{s}^{L}\right)(t)=0 \quad(t \in I) .
$$

This together with (1.2), (1.3) and (1.4) deduces

$$
\begin{aligned}
\dot{\beta}_{s}^{L}(t)= & \frac{\partial}{\partial y_{1}}+\left\{\left.\frac{\partial y_{2}}{\partial x_{1}}\right|_{\beta_{s}^{L}(t)}+\left.\frac{\partial y_{2}}{\partial x_{2}}\right|_{\beta_{s}^{L}(t)}\right. \\
& \left.\times\left(\sin \frac{1}{(t-1)^{2}}-\frac{2}{(t-1)^{2}} \cos \frac{1}{(t-1)^{2}}\right)\right\} \frac{\partial}{\partial y_{2}} \\
& +\left\{\left.\frac{\partial y_{3}}{\partial x_{1}}\right|_{\beta_{s}^{L}(t)}+\left.\frac{\partial y_{3}}{\partial x_{3}}\right|_{\beta_{s}^{L}(t)}\left(\cos \frac{1}{(t-1)^{2}}+\frac{2}{(t-1)^{2}} \sin \frac{1}{(t-1)^{2}}\right)\right\} \frac{\partial}{\partial y_{3}} \\
& +\sum_{t=r+1}^{n} \frac{d\left(x_{l} \circ \beta_{s}^{L}\right)}{d t} \frac{\partial}{\partial y_{i}} \quad(t \in I) .
\end{aligned}
$$

Since this vector belongs to $D_{\beta_{s}^{L}(t)}$, we can obtain $d\left(x_{l} \circ \beta_{s}^{L}\right) / d t=0(r+1 \leq i \leq$ $n-1)$ and

$$
\begin{aligned}
& \frac{d\left(x_{n} \circ \beta_{s}^{L}\right)}{d t} \\
= & \left(x_{n} \circ \beta_{s}^{L}\right)(t) \cdot\left(\frac{\eta_{1}^{2}\left(\partial y_{2} / \partial x_{2}\right)\left(\partial y_{3} / \partial x_{3}\right)}{\left(\eta_{1}\left(\partial y_{2} / \partial x_{2}\right)+\eta_{2}\right)\left(\eta_{1}\left(\partial y_{3} / \partial x_{3}\right)+\eta_{2}\right)}\right)\left(\beta_{s}^{L}(t)\right) \\
& \times\left\{2 \eta_{2}\left(\beta_{s}^{L}(t)\right) \cdot(t-1)\left(\cos \frac{1}{(t-1)^{2}}-\sin \frac{1}{(t-1)^{2}}\right)+\frac{2}{1-t}\right\}
\end{aligned}
$$

on I, where we use $\partial y_{i} / \partial x_{1}\left(\beta_{s}^{L}(t)\right)=2\left(\partial y_{i} / \partial x_{i}\right)\left(\beta_{s}^{L}(t)\right)(i=2,3, t \in I)$. In particular, if $\beta_{s}^{L}(t)$ is a boundary point of $U_{2}$, then $\eta_{2}\left(\beta_{s}^{L}(t)\right)=0$ and hence $\left(d\left(x_{n} \circ \beta_{s}^{L}\right) / d t\right)(t)=\left(x_{n} \circ \beta_{s}^{L}\right)(t) \times 2 /(1-t) \leq-2 /(1-t)<0$. This implies that $\beta_{s}^{L}(t) \in U_{2}$ holds for every $t \in\left(t_{1}, t_{0}\right)$. Suppose that there is $t_{2} \in\left[t_{1}, t_{0}\right)$ with $\left(x_{n} \circ \beta_{s}^{L}\right)\left(t_{2}\right) \leq-2$. Then, since $\beta_{s}^{L}\left(t_{2}\right) \in M \backslash U_{1}$ and hence $\eta_{1}\left(\beta_{s}^{L}\left(t_{2}\right)\right)=0$, by (1.7), we have $d\left(x_{n} \circ \beta_{s}^{L}\right) /\left.d t\right|_{t=t_{2}}=0$. This implies that $\left(x_{n} \circ \beta_{s}^{L}\right)(t) \geq-2$ for every $t \in\left[0, t_{0}\right)$, which furthermore implies $t_{0}=1$. That is, $\beta_{s}^{L}$ is defined over $[0,1)$. Also, we have $I=\left[t_{1}, 1\right)$. It follows from $(1.1)$ and $\left.\left(\lambda \circ x_{n}\right)\right|_{\bar{U}_{2}}=1$ that

$$
\begin{aligned}
& \frac{\partial y_{2}}{\partial x_{2}}\left(\beta_{s}^{L}(t)\right)=\left(1+\frac{1}{(1-t)\left(2+\sin \left(1 /(t-1)^{2}\right)\right)}\right) e^{-1 /\left((1-t)\left(2+\sin \left(1 /(t-1)^{2}\right)\right)\right)}>0 \\
& \frac{\partial y_{3}}{\partial x_{3}}\left(\beta_{s}^{L}(t)\right)=\left(1+\frac{1}{(1-t)\left(2+\cos \left(1 /(t-1)^{2}\right)\right)}\right) e^{-1 /\left((1-t)\left(2+\cos \left(1 /(t-1)^{2}\right)\right)\right)}>0
\end{aligned}
$$

for $t \in I=\left[t_{1}, 1\right)$. Therefore, from (1.7), (1.8), (1.9) and $\left(x_{n} \circ \beta_{s}^{L}\right)(t) \leq-1(t \in$ 
$\left.I=\left[t_{1}, 1\right)\right)$, we see that $d\left(x_{n} \circ \beta_{s}^{L}\right) / d t \leq 0$ holds on $\left[\max \left\{t_{1}, 1-1 /(\sqrt[4]{2})\right\}, 1\right)$. This together with $x_{n} \circ \beta_{s}^{L} \geq-2$ implies that $\lim _{t \rightarrow 1-0}\left(x_{n} \circ \beta_{s}^{L}\right)(t)$ exists and $\lim _{t \rightarrow 1-0}\left(x_{n} \circ \beta_{s}^{L}\right)(t)<-1$, which furthermore implies $\lim _{t \rightarrow 1-0} \pi^{\prime}\left(\beta_{s}^{L}(t)\right) \in \pi^{\prime}\left(S_{2} \cap\right.$ $\left.\pi^{-1}\left(\lim _{t \rightarrow 1-0} \beta(t)\right)\right)$. Since $\lim _{t \rightarrow 1-0} \beta_{s}^{L}(t) \in M \backslash U_{1}$, there is a sufficiently small positive number $\varepsilon$ with $\eta_{1}\left(\beta_{s}^{L}(t)\right)=0$ for $t \in[1-\varepsilon, 1)$. It follows from (1.7) that $d\left(x_{n} \circ \beta_{s}^{L}\right) / d t=0$ over $[1-\varepsilon, 1)$. This together with $x_{l} \circ \beta_{s}^{L} \equiv 0(r+1 \leq i \leq$ $n-1$ ) implies that a continuous curve $\widehat{\pi}^{\prime} \circ \beta_{s}^{L}:[0,1] \rightarrow \boldsymbol{R}^{n-r}$ defined as in (xii) is a $C^{\infty}$-curve. Thus $D$ satisfies the conditions (x)-(xii). That is, this triple $(M, \mathfrak{F}, D)$ admits a singular non-extendable rectangle without terminal vertex. In this example, it is sufficient that $r \geq 3$ and $n \geq r+1$. Therefore, Theorem 1 has been proved.

It is natural to consider the following problem.

Problem. Is there a triple $(M, \mathfrak{F}, D)$ admitting a singular non-extendable rectangle without terminal vertex for $r=2$ and $n \geq 3$ ?

\section{Proof of Theorem 2}

In this section, we shall prove Theorem 2 by investigating a property of a singular non-extendable rectangle without terminal vertex. First we prepare the following lemma.

LEMMA. Let $\mathfrak{F}$ be a foliation on a Riemannian manifold $(M, g)$ and take the orthogonal complementary distribution $F^{\perp}$ of $\mathfrak{F}$ as a complementary distribution to $\mathfrak{F}$. If $\delta$ is a singular non-extendable rectangle without terminal vertex, then $\lim _{t \rightarrow 1-0} l\left(\left.\delta_{\cdot 1}\right|_{[0, t]}\right)=\infty$ holds.

Proof. Set $p_{0}:=\lim _{t \rightarrow 1-0} \delta(t, 1)$. Take a foliated coordinate neighbourhood $\left(\tilde{U}, \tilde{\phi}=\left(x_{1}, \ldots, x_{n}\right)\right)$ around $p_{0}$ with $\tilde{\phi}\left(p_{0}\right)=(0, \ldots, 0)$ and $\tilde{\phi}(\tilde{U})=(-2,2)^{n}$, where $n=\operatorname{dim} M$ and the foliatedness of $(\tilde{U}, \tilde{\phi})$ implies that fibres of the submersion $\pi:=\left(x_{1}, \ldots, x_{r}\right): \tilde{U} \rightarrow \boldsymbol{R}^{r}(r=\operatorname{codim} \mathfrak{F})$ are leaves of $\left.\mathfrak{F}\right|_{\tilde{U}}$. Let $D$ be a complementary distribution to $\mathfrak{F}$ on $\tilde{U}$ spanned by $\partial / \partial x_{1}, \ldots, \partial / \partial x_{r}$. Denote by $L_{p_{0}}^{V}$ the leaf of $\left.\mathfrak{F}\right|_{\tilde{U}}$ through $p_{0}$ and $L_{p_{0}}^{D}$ the maximal integral manifold of $D$ through $p_{0}$. Let $\pi_{V}: \tilde{U} \rightarrow L_{p_{0}}^{V}$ (resp. $\pi_{D}: \tilde{U} \rightarrow L_{p_{0}}^{D}$ ) be the projection whose fibres are the maximal integral manifolds of $D$ (resp. leaves of $\left.\mathfrak{F}\right|_{\tilde{U}}$ ). Give $\tilde{U}$ a flat Riemannian metric $g_{0}$ defined by $g_{0}\left(\partial / \partial x_{l}, \partial / \partial x_{j}\right)=\delta_{i j}(i, j=1, \ldots, n)$ and denote by $d_{0}$ the distance function induced from $g_{0}$, where $\delta_{i j}$ is the Kronecker's delta. Set $U:=\tilde{\phi}^{-1}\left((-1,1)^{n}\right)$. Take increasing sequences $\left\{t_{k}\right\}_{k=1}^{\infty}$ and $\left\{s_{k}\right\}_{k=1}^{\infty}$ in $[0,1)$ satisfying $\lim _{k \rightarrow \infty} t_{k}=\lim _{k \rightarrow \infty} s_{k}=1, \quad \delta_{.1}\left(\left[t_{k}, 1\right)\right) \cup \delta_{t_{k}}\left(\left[s_{k}, 1\right]\right) \subset U$, $\max _{t \in\left[t_{k}, 1\right)} d_{0}\left(\pi_{D}(\delta(t, 1)), p_{0}\right)<1 / k$ and $\max _{s \in\left[s_{k}, 1\right]} d_{0}\left(\pi_{V}\left(\delta\left(t_{k}, s\right)\right), p_{0}\right)<1 / k$ (see Figure 2.1). 


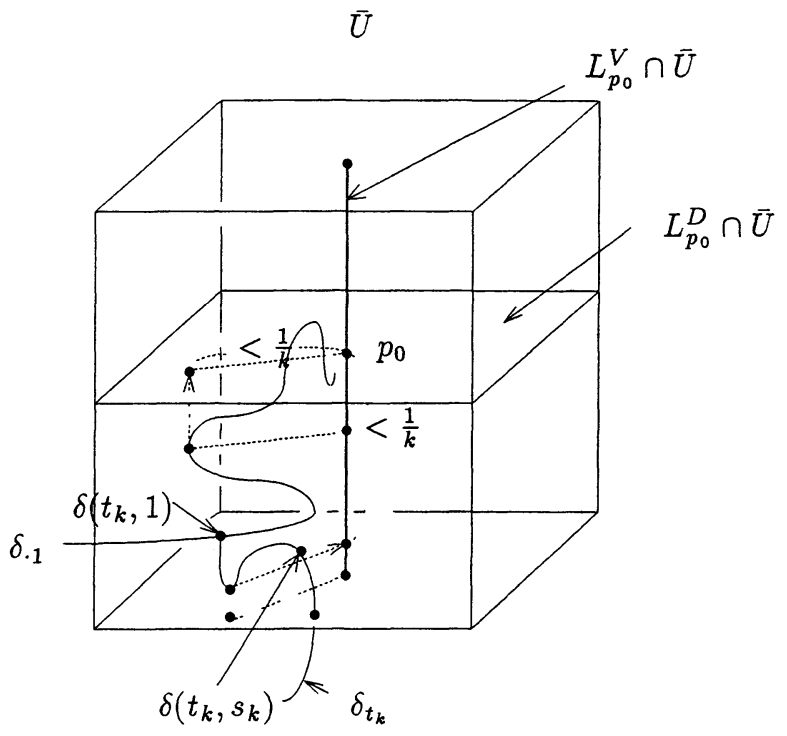

Figure 2.1.

We can show that $\delta_{s_{k}}\left(\left[t_{k}, 1\right]\right)$ is not contained in $\tilde{U}$ for every $k$. In fact, if $\delta_{s_{k}}\left(\left[t_{k}, 1\right]\right) \subset \tilde{U}$ for some $k$, then the existence of the $F^{\perp}$-lift of $\left.\pi \circ \delta \cdot s_{k}\right|_{\left[t_{k}, 1\right]}$ starting from $\delta\left(t_{k}, 1\right)$ is assured because $\lim _{t \rightarrow 1-0} \delta \delta_{1}(t)=p_{0} \in \tilde{U}$ and hence $\delta$ is extendable. This contradicts the fact that $\delta$ is non-extendable. Thus $\delta \cdot s_{k}\left(\left[t_{k}, 1\right]\right)$ $\not \subset \tilde{U}$ for every $k$. That is, $\delta_{s_{k}}\left(\left[t_{k}, 1\right]\right) \cap \partial U \neq \emptyset$ holds for every $k$, where $\partial U$ is the boundary of $U$ in $M$. Set $t_{k}^{\prime}:=\min \left\{t \in\left[t_{k}, 1\right] \mid \delta_{\cdot s_{k}}(t) \in \partial U\right\}$. Take $\left(t_{k}^{\prime \prime}, s_{k}^{\prime}\right) \in$ $\left(t_{k}, t_{k}^{\prime}\right] \times\left[s_{k}, 1\right)$ satisfying $\delta\left(\left[t_{k}, t_{k}^{\prime \prime}\right] \times\left[s_{k}^{\prime}, 1\right]\right) \cap \partial U=\left\{\delta\left(t_{k}^{\prime \prime}, s_{k}^{\prime}\right)\right\}$ (see Figure 2.2).

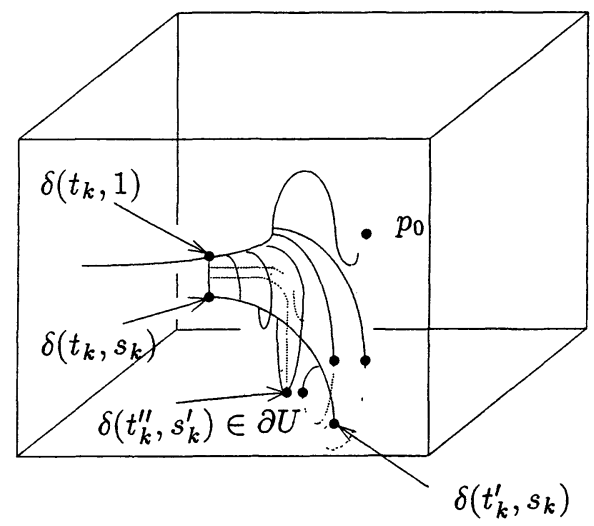

Figure 2.2. 
Denote by $S(\bar{U})$ the unit tangent bundle of $\bar{U}$ with respect to $g_{0}$ and $F$ the tangent bundle of $\mathfrak{F}$, where $\bar{U}$ is the closure of $U$. Define $\beta_{k}:\left[t_{k}, t_{k}^{\prime \prime}\right] \rightarrow M$ by $\beta_{k}(t):=\delta_{s_{k}^{\prime}}(t)$ for $t \in\left[t_{k}, t_{k}^{\prime \prime}\right]$ and $X_{k}(t):=\dot{\beta}_{k}(t) /\left\|\dot{\beta}_{k}(t)\right\| \in S(\bar{U})$, where $\left\|\dot{\beta}_{k}(t)\right\|=$ $\sqrt{g_{0}\left(\dot{\beta}_{k}(t), \dot{\beta}_{k}(t)\right)}$. Take $t_{k}^{\prime \prime \prime} \in\left[t_{k}, t_{k}^{\prime \prime}\right]$ such that

$$
\frac{\left\|X_{k}\left(t_{k}^{\prime \prime \prime}\right)^{V}\right\|}{\left\|X_{k}\left(t_{k}^{\prime \prime \prime}\right)^{D}\right\|}=\max _{t \in\left[t_{k}, t_{k}^{\prime \prime}\right]} \frac{\left\|X_{k}(t)^{V}\right\|}{\left\|X_{k}(t)^{D}\right\|},
$$

where $X_{k}(t)^{V}$ (resp. $\left.X_{k}(t)^{D}\right)$ is the $F$-component (resp. the $D$-component) of $X_{k}(t)$. It follows from the compactness of $S(\bar{U})$ that there is a convergent subsequence $\left\{Y_{k}\right\}_{k=1}^{\infty}$ of $\left\{X_{k}\left(t_{k}^{\prime \prime \prime}\right)\right\}_{k=1}^{\infty}$. Set $Y_{0}:=\lim _{k \rightarrow \infty} Y_{k}$. Since $Y_{k} \in F^{\perp}$ for every $k$, we have $Y_{0} \in F^{\perp}$. Suppose $\lim _{k \rightarrow \infty}\left\|X_{k}\left(t_{k}^{\prime \prime \prime}\right)^{V}\right\| /\left\|X_{k}\left(t_{k}^{\prime \prime \prime}\right)^{D}\right\|=\infty$. Then, we have $\lim _{k \rightarrow \infty}\left\|Y_{k}^{V}\right\| /\left\|Y_{k}^{D}\right\|=\infty$, which implies $Y_{0} \in F$. Thus $Y_{0} \in F \cap F^{\perp}$, that is, $Y_{0}=0$ is deduced. This contradicts $Y_{0} \in S(\bar{U})$. Therefore, $\lim _{k \rightarrow \infty}\left\|X_{k}\left(t_{k}^{\prime \prime \prime}\right)^{V}\right\| /$ $\left\|X_{k}\left(t_{k}^{\prime \prime \prime}\right)^{D}\right\|=\infty$ does not hold. Hence, for a sufficiently large positive number $c$, there is a subsequence $\left\{X_{a(k)}\left(t_{a(k)}^{\prime \prime \prime}\right)\right\}_{k=1}^{\infty}$ of $\left\{X_{k}\left(t_{k}^{\prime \prime \prime}\right)\right\}_{k=1}^{\infty}$ such that $\left\|X_{a(k)}\left(t_{a(k)}^{\prime \prime \prime}\right)^{V}\right\| /$ $\left\|X_{a(k)}\left(t_{a(k)}^{\prime \prime \prime}\right)^{D}\right\|<c$ for every $k$. Since $\left\|X_{a(k)}(t)^{V}\right\| /\left\|X_{a(k)}(t)^{D}\right\|<c\left(t \in\left[t_{a(k)}, t_{a(k)}^{\prime \prime}\right]\right)$ by the definition of $t_{a(k)}^{\prime \prime \prime}$, we have

$$
l_{0}\left(\pi_{D} \circ \beta_{a(k)}\right)>\frac{1}{c} l_{0}\left(\pi_{V} \circ \beta_{a(k)}\right)
$$

where $l_{0}(\cdot)$ is the length of a curve - with respect to $g_{0}$. Also, it follows from $\max _{s \in\left[s_{a(k)}, 1\right]} d_{0}\left(\pi_{V}\left(\delta\left(t_{a(k)}, s\right)\right), p_{0}\right)<1 / a(k)$ and $\delta\left(t_{a(k)}^{\prime \prime}, s_{a(k)}^{\prime}\right) \in \partial U$ that $d_{0}\left(\pi_{V}\left(\delta\left(t_{a(k)}, s_{a(k)}^{\prime}\right)\right), p_{0}\right)<1 / a(k)$ and $d_{0}\left(\pi_{V}\left(\delta\left(t_{a(k)}^{\prime \prime}, s_{a(k)}^{\prime}\right)\right), p_{0}\right) \geq 1$, respectively. Hence we have

$$
\begin{aligned}
l_{0}\left(\pi_{V} \circ \beta_{a(k)}\right) & >d_{0}\left(\pi_{V}\left(\delta\left(t_{a(k)}, s_{a(k)}^{\prime}\right)\right), \pi_{V}\left(\delta\left(t_{a(k)}^{\prime \prime}, s_{a(k)}^{\prime}\right)\right)\right) \\
& \geq d_{0}\left(\pi_{V}\left(\delta\left(t_{a(k)}^{\prime \prime}, s_{a(k)}^{\prime}\right)\right), p_{0}\right)-d_{0}\left(\pi_{V}\left(\delta\left(t_{a(k)}, s_{a(k)}^{\prime}\right)\right), p_{0}\right) \\
& >1-\frac{1}{a(k)} \\
& \geq 1-\frac{1}{k} .
\end{aligned}
$$

Since $\left.\delta_{t}\right|_{\left[s_{a(k)}^{\prime}, 1\right]}\left(t \in\left[t_{a(k)}, t_{a(k)}^{\prime \prime}\right]\right)$ are vertical curves in $U$ by $\delta\left(\left[t_{a(k)}, t_{a(k)}^{\prime \prime}\right] \times\right.$ $\left.\left[s_{a(k)}^{\prime}, 1\right]\right) \cap \partial U=\left\{\delta\left(t_{a(k)}^{\prime \prime}, s_{a(k)}^{\prime}\right)\right\}$, we have $\left.\pi_{D} \circ \delta_{1}\right|_{\left[t_{a(k)}, t_{a(k)}^{\prime \prime}\right]}=\pi_{D} \circ \beta_{a(k)}$. Therefore, it follows from $(2.1)$ and $(2.2)$ that $l_{0}\left(\left.\pi_{D} \circ \delta_{.1}\right|_{\left[t_{a(k)}, t_{a(k)}^{\prime \prime}\right)}\right) \geq(1 / c)(1-1 / k)$. We may assume that $t_{k}^{\prime \prime} \leq t_{k+1}$ holds for every $k$ by retaking $\left\{t_{k}\right\}_{k=1}^{\infty}$ if necessary. Hence we obtain 


$$
\begin{aligned}
\lim _{t \rightarrow 1-0} l_{0}\left(\left.\delta \cdot 1\right|_{\left[t_{a(1)}, t\right]}\right) & \geq \lim _{t \rightarrow 1-0} l_{0}\left(\left.\pi_{D} \circ \delta \cdot 1\right|_{\left[t_{a(1)}, t\right]}\right) \\
& \geq \sum_{k=1}^{\infty} l_{0}\left(\left.\pi_{D} \circ \delta \cdot 1\right|_{\left[t_{a(k)}, t_{a(k)}^{\prime \prime}\right)}\right) \\
& \geq \sum_{k=1}^{\infty} \frac{1}{c}\left(1-\frac{1}{k}\right) \\
& =\infty .
\end{aligned}
$$

Define a function $\rho$ on the projective bundle $\operatorname{Pr}(T \tilde{U})$ of $T \tilde{U}$ by

$$
\rho(W):=\sqrt{\frac{g(X, X)}{g_{0}(X, X)}}
$$

for $W \in \operatorname{Pr}(T \tilde{U})$, where $X$ is a non-zero vector belonging to $W$. It is clear that $\rho$ is continuous. Since $\delta_{._{1}}\left(\left[t_{a(1)}, 1\right)\right) \cup\left\{p_{0}\right\}$ is compact, so is also

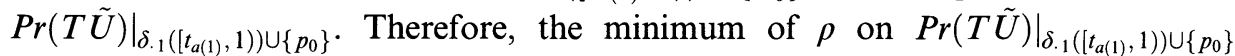
exists. Denote by $c^{\prime}$ this minimum. Clearly we have $c^{\prime}>0$. Then, it is easy to show that $l\left(\left.\delta_{1}\right|_{\left[t_{a(1)}, t\right]}\right) \geq c^{\prime} l_{0}\left(\left.\delta_{.1}\right|_{\left[t_{a(1)}, t\right]}\right)$ for every $t \in\left[t_{a(1)}, 1\right)$. Therefore, we obtain

$$
\lim _{t \rightarrow 1-0} l\left(\left.\delta_{1}\right|_{\left[t_{a(1)}, t\right]}\right)=c^{\prime} \lim _{t \rightarrow 1-0} l_{0}\left(\left.\delta_{\cdot 1}\right|_{\left[t_{a(1)}, t\right]}\right)=\infty,
$$

that is, $\lim _{t \rightarrow 1-0} l\left(\left.\delta_{1}\right|_{[0, t]}\right)=\infty$.

Now we shall prove Theorem 2 in terms of this lemma.

Proof of Theorem 2. Suppose that $F^{\perp}$ is not an Ehresmann connection. Then there is a non-extendable rectangle $\delta$ without terminal vertex. If $\delta$ is nonsingular, then $\lim _{t \rightarrow 1-0} l\left(\left.\delta_{1}\right|_{[0, t]}\right)=\infty$ is deduced from the completeness of $(M, g)$. Also, if $\delta$ is singular, then $\lim _{t \rightarrow 1-0} l\left(\left.\delta_{1}\right|_{[0, t]}\right)=\infty$ is deduced from Lemma. Whether $\delta$ is non-singular or not, we obtain $\lim _{t \rightarrow 1-0} l\left(\left.\delta_{.1}\right|_{[0, t]}\right)=\infty$. This deduces $\lim _{t \rightarrow 1-0} G_{\delta_{0}}^{\perp}\left(\left.\delta\right|_{[0, t] \times[0,1]}\right)=\infty$. Hence sup $G_{\delta_{0}}^{\perp}<\infty$ does not hold, which contradicts the assumption. Therefore, $F^{\perp}$ is an Ehresmann connection.

\section{Examples}

In this section, we shall first give examples of non-singular non-extendable rectangles without terminal vertex.

EXAMPLE 1. Let $B:=\left\{\left(x_{1}, \ldots, x_{n}\right) \in \boldsymbol{R}^{n} \mid x_{1}^{2}+\cdots+x_{n}^{2}<1\right\} \quad(n \geq 2)$ and $\mathfrak{F}$ a foliation of codimension $r$ on $B$ whose leaves are fibres of the projection $\pi$ : $B \rightarrow \boldsymbol{R}^{r}$ defined by $\pi\left(x_{1}, \ldots, x_{n}\right)=\left(x_{1}, \ldots, x_{r}\right)$ for $\left(x_{1}, \ldots, x_{n}\right) \in B$, where $1 \leq r \leq$ $n-1$. Let $D=\operatorname{Span}\left\{\partial / \partial x_{1}, \ldots, \partial / \partial x_{r}\right\}$, where we regard $\left(x_{1}, \ldots, x_{n}\right)$ as a co- 
ordinate of $B$. Define a rectangle $\delta$ without terminal vertex by $\delta(t, s):=(t / \sqrt{2}$, $0, \ldots, 0, s / \sqrt{2}$ ). It is clear that $\delta$ is non-extendable and non-singular (see Figure $3.1)$.

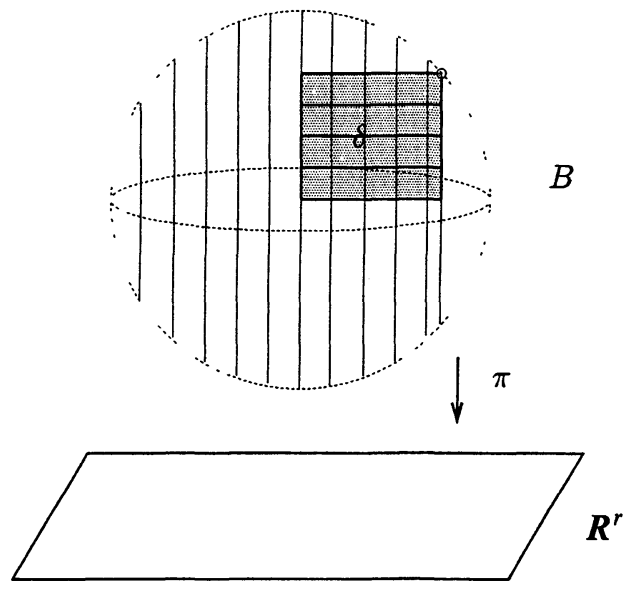

Figure 3.1.

EXAMPLE 2. Let $\mathfrak{F}$ be a foliation of codimension one on an $n$-dimensional affine space $\boldsymbol{R}^{n}(n \geq 2)$ whose leaves are

$$
\left\{\left(x_{1}, \ldots, x_{n-1}, k-\exp \left(1-\sum_{l=1}^{n-1} x_{l}^{2}\right)^{-1} \mid \sum_{l=1}^{n-1} x_{l}^{2}<1\right\} \quad(k \in \boldsymbol{R})\right.
$$

and

$$
\left\{\left(x_{1}, \ldots, x_{n}\right) \mid \sum_{l=1}^{n-1} x_{i}^{2}=k^{2}, x_{n} \in \boldsymbol{R}\right\} \quad(k \geq 1) .
$$

Let $D$ be the orthogonal complementary distribution of $\mathfrak{F}$ with respect to the Euclidean metric $g$ of $\boldsymbol{R}^{n}$ defined by $g\left(\partial / \partial x_{l}, \partial / \partial x_{J}\right)=\delta_{i j}$, where we regard $\left(x_{1}, \ldots, x_{n}\right)$ as a coordinate of $\boldsymbol{R}^{n}$ and $\delta_{i j}$ is the Kronecker's delta. Let $\alpha$ be a vertical curve defined by $\alpha(s)=\left((1-s) / 2,0, \ldots, 0, k-e^{4 /((3-s)(1+s))}\right)$ for $s \in[0,1]$ and $\beta$ be a horizontal curve defined by $\beta(t)=((1+t) / 2,0, \ldots, 0$, $\left.(1 / 32) \int_{0}^{t}\left(\left((t+3)^{2}(t-1)^{2}\right) /(t+1)\right) e^{4 /((t+3)(t-1))} d t+k-e^{4 / 3}\right)$ for $t \in[0,1]$. It is clear that there is a rectangle $\delta$ without terminal vertex satisfying $\delta_{0}=\alpha$ and $\delta_{.0}=\beta$ but it is non-extendable and non-singular (see Figure 3.2). 


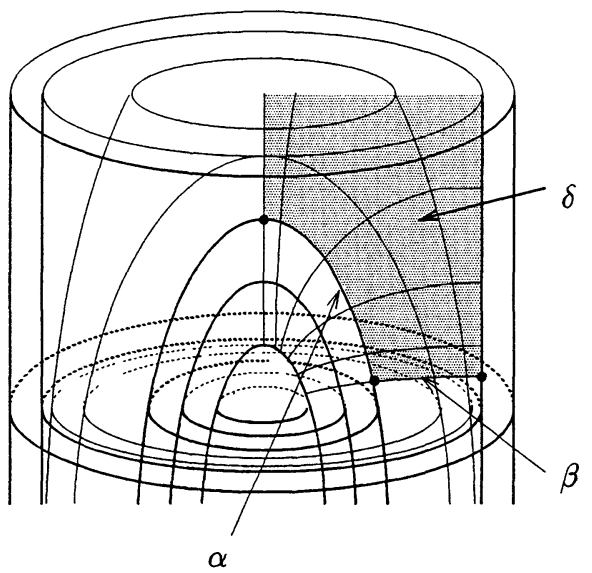

Figure 3.2.

Next we shall give examples of a foliated Riemannian manifold which satisfies the assumption of Theorem 2 but does not satisfy the condition (IV) in Introduction.

EXAMPLE 3. Let $M$ be a hypersurface of an $(n+1)$-dimensional Euclidean space $\boldsymbol{R}^{n+1}(n \geq 2)$ defined by the equation $x_{1}^{2}+\cdots+x_{r+1}^{2}-x_{r+2}^{2}-\cdots-x_{n+1}^{2}=$ $1(1 \leq r \leq n-1)$ and give $M$ the Riemannian metric $g$ induced from the Euclidean metric of $\boldsymbol{R}^{n+1}$, where $\left(x_{1}, \ldots, x_{n+1}\right)$ is a Euclidean coordinate system of $\boldsymbol{R}^{n+1}$. It is clear that $(M, g)$ is complete. Let $\mathfrak{F}$ be a foliation on $(M, g)$ whose leaves are the intersections of $M$ and $(n-r+1)$-dimensional halfplanes

$$
\begin{aligned}
& \left\{\left(t c_{1}, \ldots, t c_{r+1}, x_{r+2}, \ldots, x_{n+1}\right) \mid\left(x_{r+2}, \ldots, x_{n+1}\right) \in \boldsymbol{R}^{n-r}, t \geq 0\right\} \\
& \left(\left(c_{1}, \ldots, c_{r+1}\right) \in S^{r}(1)=\left\{\left(x_{1}, \ldots, x_{r+1}\right) \mid x_{1}^{2}+\cdots+x_{r+1}^{2}=1\right\}\right) .
\end{aligned}
$$

Then the orthogonal complementary distribution $F^{\perp}$ of $\mathfrak{F}$ is an integrable distribution whose maximal integral manifolds are the intersections of $M$ and $(r+1)$-dimensional planes

$$
\begin{gathered}
\left\{\left(x_{1}, \ldots, x_{r+1}, c_{r+2}, \ldots, c_{n+1}\right) \mid\left(x_{1}, \ldots, x_{r+1}\right) \in \boldsymbol{R}^{r+1}\right\} \\
\left(\left(c_{r+2}, \ldots, c_{n+1}\right) \in \boldsymbol{R}^{n-r}\right) .
\end{gathered}
$$

It is shown that $G_{\alpha}^{\perp} \equiv \sqrt{\alpha_{1}(1)^{2}+\cdots+\alpha_{r+1}(1)^{2}} / \sqrt{\alpha_{1}(0)^{2}+\cdots+\alpha_{r+1}(0)^{2}}$ holds for each vertical curve $\alpha$ (see Figure 3.3), where $\alpha=\left(\alpha_{1}, \ldots, \alpha_{n+1}\right)$. 


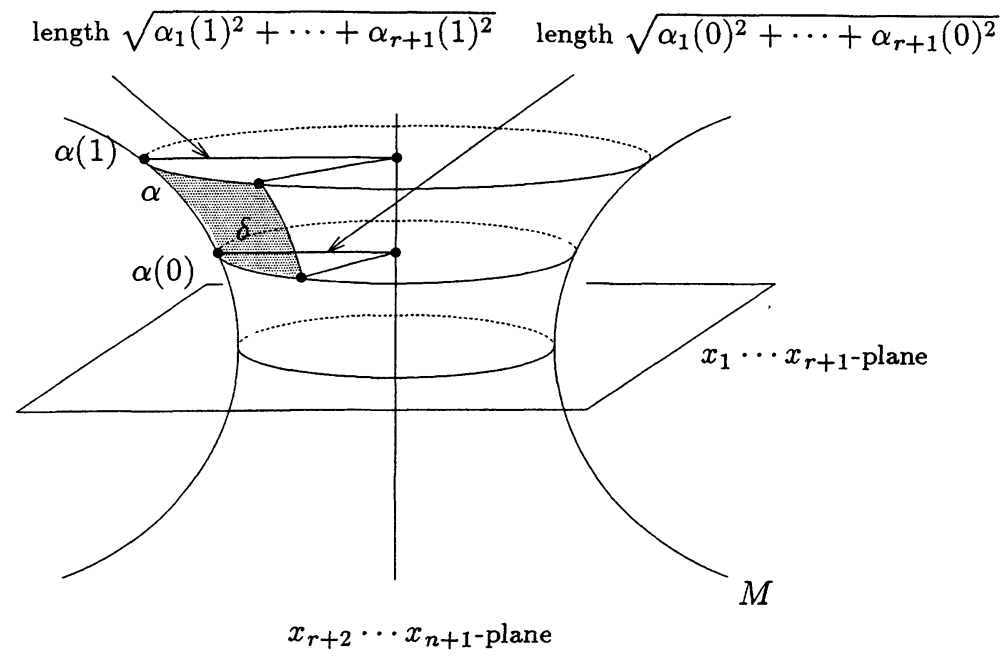

Figure 3.3.

Thus $\mathfrak{F}$ satisfies the assumption of Theorem 2 . Let $\alpha_{0}$ be a vertical curve without terminal point defined by $\alpha_{0}(s):=\left(1 /(1-s), 0, \ldots, 0, \sqrt{2 s-s^{2}} /(1-s)\right)$ for $s \in[0,1)$. From $G_{\left.\alpha_{0}\right|_{[0, s]}}^{\perp} \equiv \sqrt{\left(\alpha_{0}\right)_{1}(s)^{2}+\cdots+\left(\alpha_{0}\right)_{r+1}(s)^{2}} / \sqrt{\left(\alpha_{0}\right)_{1}(0)^{2}+\cdots+\left(\alpha_{0}\right)_{r+1}(0)^{2}}=1 /$ $(1-s)$, we have $\lim _{s \rightarrow 1-0} \sup G_{\alpha_{0} \mid[0, s]}^{\perp}=\infty$ and hence $\sup _{s \in[0,1)} \sup G_{\left.\alpha_{0}\right|_{[0, s]}}^{\perp}<\infty$ does not hold. Thus $\mathfrak{F}$ does not satisfy the condition (IV).

In this example, the base manifold $M$ is not compact. Next we shall give an example such that the base manifold is compact.

EXAMPLE 4. Let $\tilde{\mathbb{F}}$ be a foliation on a 2-dimensional Euclidean space $\boldsymbol{R}^{2}$ whose leaves are

$$
\left\{\left(x_{1}, \tan x_{1}+c\right) \mid \frac{(2 k-1) \pi}{2}<x_{1}<\frac{(2 k+1) \pi}{2}\right\} \quad(c \in \boldsymbol{R}, k \in \boldsymbol{N})
$$

and

$$
\left\{\left(\frac{(2 k+1) \pi}{2}, x_{2}\right) \mid x_{2} \in \boldsymbol{R}\right\} \quad(k \in N) .
$$

Let $\phi_{1}$ be a translation of $\boldsymbol{R}^{2}$ defined by $\phi_{1}\left(x_{1}, x_{2}\right)=\left(x_{1}+\pi, x_{2}\right)$ for $\left(x_{1}, x_{2}\right) \in \boldsymbol{R}^{2}$ and $\phi_{2}$ a translation of $\boldsymbol{R}^{2}$ defined by $\phi_{2}\left(x_{1}, x_{2}\right)=\left(x_{1}, x_{2}+1\right)$ for $\left(x_{1}, x_{2}\right) \in \boldsymbol{R}^{2}$. Denote by $G$ the transformation group of $\boldsymbol{R}^{2}$ generated by $\phi_{1}$ and $\phi_{2}$. Denote by $M$ the orbit space $\boldsymbol{R}^{2} / G$ of $G$. Since $G$ is an isometry group of $\boldsymbol{R}^{2}$, the Euclidean metric $\tilde{g}$ of $\boldsymbol{R}^{2}$ induces a Riemannian metric on $M$, which we denote by $g$. Also, since $G$ preserves $\tilde{\mathfrak{F}}$, $\tilde{\mathfrak{F}}$ induces a foliation on $M$, which we denote by $\mathfrak{F}$. Denote by $\tilde{F}^{\perp}$ (resp. $F^{\perp}$ ) the orthogonal complementary distribution of $\tilde{\mathscr{F}}$ 
(resp. $\mathfrak{F}$ ) and $\tilde{\mathfrak{F}}^{\perp}$ (resp. $\mathfrak{F}^{\perp}$ ) the foliation whose leaves are the maximal integral manifolds of $\tilde{F}^{\perp}$ (resp. $\left.F^{\perp}\right)$. Denote by $\Gamma(\cdot)$ the space of all cross sections of a vector bundle $\cdot$ We define $h \in \Gamma\left(\left(\tilde{F}^{\perp}\right)^{*} \otimes\left(\tilde{F}^{\perp}\right)^{*} \otimes \tilde{F}\right)$ by $h(X, Y):=\left(\tilde{\nabla}_{X} Y\right)_{\tilde{F}}$ for $X, Y \in \Gamma\left(\tilde{F}^{\perp}\right)$, where $\tilde{\nabla}$ is the Levi-Civita connection of $\tilde{g}$ and $\left(\tilde{\nabla}_{X} Y\right)_{\tilde{F}}$ is the $\tilde{F}$ component of $\tilde{\nabla}_{X} Y$. Let $S \tilde{F}^{\perp}$ (resp. $S \tilde{F}$ ) be a sphere bundle consisting of all unit vectors belonging to $\tilde{F}^{\perp}$ (resp. the tangent bundle $\tilde{F}$ of $\tilde{F}$ ). Then, it is easy to show that $\|h(X, X)\|<1$ holds for every $X \in S \tilde{F}^{\perp}$, where $\|h(X, X)\|$ is the norm of $h(X, X)$ (see Fig. 3.4). Also, it is shown that $\left\|P_{\tilde{\beta}}^{B} Y\right\| \leq \sqrt{2}$ holds for every horizontal curve $\tilde{\beta}$ in $\boldsymbol{R}^{2}$ and every $Y \in S \tilde{F}_{\tilde{\beta}(0)}$, where $P_{\tilde{\beta}}^{B}$ is the parallel translation along $\tilde{\beta}$ with respect to the Bott connection on ${ }^{\beta}$ the orthogonal complementary distribution $F$ of $\tilde{\mathfrak{F}}^{\perp}$ (see Figure 3.4).

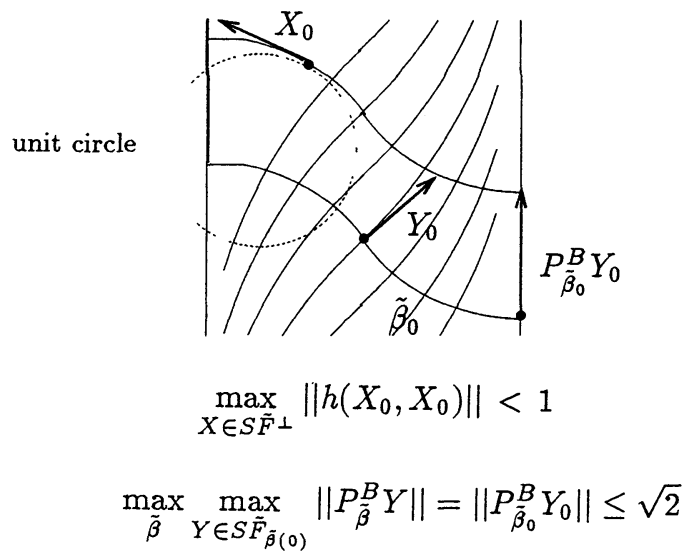

Figure 3.4.

Therefore, we can obtain

$$
\sup _{\tilde{\beta}: \text { honzontal curve }} \sup _{X \in S F_{\tilde{\beta}(1)}^{\perp}, Y \in S F_{\tilde{\beta}(0)}}\left|\tilde{g}\left(P_{\tilde{\beta}}^{B} Y, h(X, X)\right)\right| \leq \sqrt{2}<\infty .
$$

Set

$$
A:=\sup _{\tilde{\beta} \text {.horzontal curve }} \sup _{X \in S F_{\tilde{\beta}(1)}^{\perp}, Y \in S F_{\tilde{\beta}(0)}}\left|\tilde{g}\left(P_{\tilde{\beta}}^{B} Y, h(X, X)\right)\right| .
$$

Then we can show that $\sup G_{\tilde{\alpha}}^{\perp} \leq \exp (A \cdot l(\tilde{\alpha}))$ holds for every vertical curve $\tilde{\alpha}$ in $\boldsymbol{R}^{2}$, where $l(\tilde{\alpha})$ is the length of $\tilde{\alpha}$ with respect to $\tilde{g}$ (see the proof of Corollary 3.10 in [11]). Take an arbitrary vertical curve $\alpha$ in $M$ and an arbitrary rectangle $\delta$ with $\delta_{0}=\alpha$. Let $\alpha^{L}$ be one of lifts of $\alpha$ to $\boldsymbol{R}^{2}$ and $\delta^{L}$ the lift of $\delta$ to $\boldsymbol{R}^{2}$ with $\delta_{0}^{L}=\alpha^{L}$. Clearly we have $G_{\alpha}^{\perp}(\delta)=G_{\alpha^{L}}^{\perp}\left(\delta^{L}\right)$, which implies $\sup G_{\alpha}^{\perp} \leq \sup G_{\alpha^{L}}^{\perp}$ by 
the arbitrariness of $\delta$. Therefore, we can obtain

$$
\sup G_{\alpha}^{\perp} \leq \sup G_{\alpha^{L}}^{\perp} \leq \exp \left(A \cdot l\left(\alpha^{L}\right)\right)=\exp (A \cdot l(\alpha)),
$$

where $l(\alpha)$ is the length of $\alpha$ with respect to $g$. Thus $\mathfrak{F}$ satisfies the assumption of Theorem 2. Define a vertical curve $\tilde{\alpha}_{0}$ in $\boldsymbol{R}^{2}$ without terminal point by $\tilde{\alpha}_{0}(s):=(-\pi / 2,1 /(1-s))$ for $s \in[0,1)$ and let $\tilde{\beta}_{0}$ be the horizontal curve in $\boldsymbol{R}^{2}$ satisfying $\tilde{\beta}_{0}(0)=\tilde{\alpha}_{0}(0)$ and $\left(\tilde{\beta}_{0}\right)_{1}(t)=t-(\pi / 2)(t \in[0,1])$, where $\tilde{\beta}_{0}=\left(\left(\tilde{\beta}_{0}\right)_{1},\left(\tilde{\beta}_{0}\right)_{2}\right)$. Take a sequence $\left\{t_{k}\right\}_{k=1}^{\infty}$ in $(0,1]$ satisfying $l\left(\left.\tilde{\beta}_{0}\right|_{\left[0, t_{k}\right]}\right)<1 / k(k \geq 1)$. For each

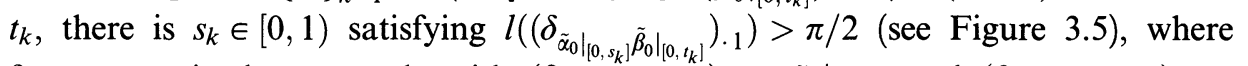
$\delta_{\left.\left.\tilde{\alpha}_{0}\right|_{\left[0, s_{k}\right]} \tilde{\beta}_{0}\right|_{\left[0, t_{k}\right]}}$ is the rectangle with $\left(\delta_{\left.\left.\tilde{\alpha}_{0}\right|_{\left[0, s_{k}\right]} \tilde{\beta}_{0}\right|_{\left.\mid 0, t_{k}\right]}}\right)_{0 .}=\left.\tilde{\alpha}_{0}\right|_{\left[0, s_{k}\right]}$ and $\left(\delta_{\left.\left.\tilde{\alpha}_{0}\right|_{\left[0, s_{k}\right]} \tilde{\beta}_{0}\right|_{\left[0, t_{k}\right]}}\right)_{.0}=$ $\left.\tilde{\beta}_{0}\right|_{\left[0, t_{k}\right]}$. Then we have

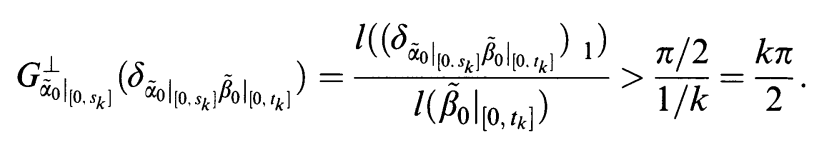

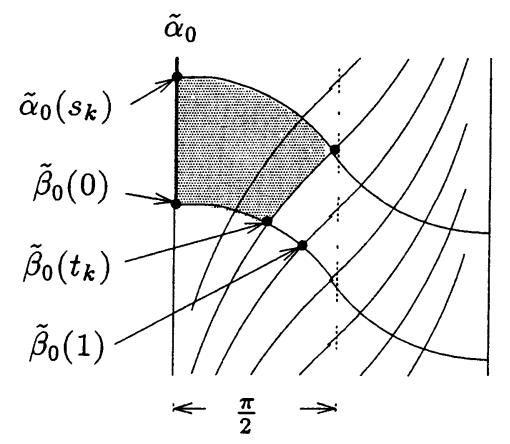

Figure 3.5.

Set $\alpha_{0}:=\pi \circ \tilde{\alpha}_{0}$ and $\beta_{0}:=\pi \circ \tilde{\beta}_{0}$, where $\pi$ is the projection of $\boldsymbol{R}^{2}$ onto $M$. From $G_{\left.\alpha_{0}\right|_{\left[0 s_{k}\right]}}^{\perp}\left(\delta_{\left.\left.\alpha_{0}\right|_{\left[0, s_{k} \mid\right.} \beta_{0}\right|_{\left[0 . t_{k}\right]}}\right)=G_{\left.\tilde{\alpha}_{0}\right|_{\left[0, s_{k}\right]}}^{\perp}\left(\delta_{\left.\left.\tilde{\alpha}_{0}\right|_{\left[0, s_{k} \mid\right.} \tilde{\beta}_{0}\right|_{\left[0, t_{k}\right]}}\right)$, we have $\lim _{k \rightarrow \infty} G_{\left.\alpha_{0}\right|_{\left[0, s_{k}\right]}}^{\perp}\left(\delta_{\left.\left.\alpha_{0}\right|_{\left[0, s_{k}\right.} \beta_{0}\right|_{\left[0, t_{k}\right]}}\right)$ $=\lim _{k \rightarrow \infty} k \pi / 2=\infty$ and hence $\sup _{s \in[0,1)} \sup G_{\left.\alpha_{0}\right|_{[0 . s]} ^{\perp}}^{\perp}<\infty$ does not hold. Thus $\mathfrak{F}$ does not satisfy the condition (IV).

From these examples, it is guessed that there are a lots of examples of a foliated Riemannian manifold which satisfies the assumption of Theorem 2 but does not satisfy the condition (IV). Thus we can recognize the essential gap between the assumption of Theorem 2 and the condition (IV).

Next we shall give examples showing the topological gap between Riemannian foliations (i.e., foliations admitting a bundlelike metric) and foliations admitting a Riemannian metric satisfying the assumption of Theorem 2 .

Example 5. Let $(M, \mathfrak{F})$ be a foliated manifold in Example 4. The above Riemannian metric $g$ satisfies the assumption of Theorem 2. However, $\mathfrak{F}$ is not 
a Riemannian foliation. In fact, for an arbitrary Riemannian metric on $M$, $G_{\alpha_{0}}^{\perp}\left(\delta_{0}\right)>1$ holds, where $\alpha_{0}$ and $\delta_{0}$ are as in Figure 3.6.

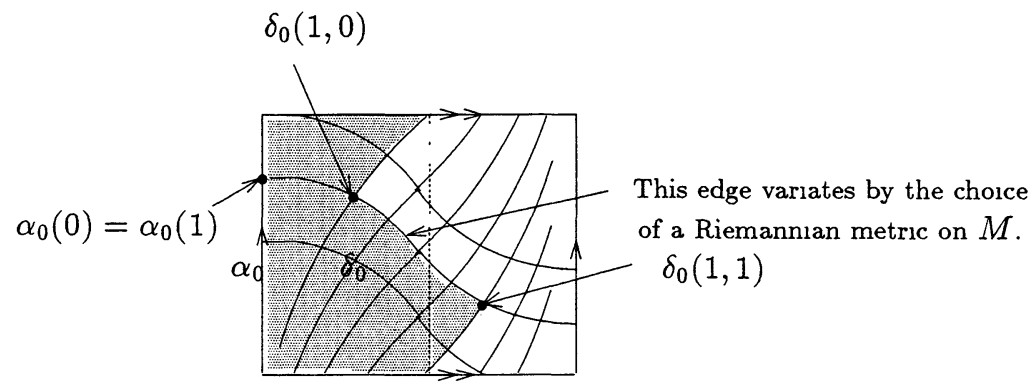

Figure 3.6.

EXAMPLE 6. Let $p_{0}$ be a point of the $n$-dimensional unit sphere $S^{n}(1)$ and $q_{0}$ the antipodal point of $p_{0}$, where we give $S^{n}(1)$ the standard Riemannian metric. Denote by $g_{1}$ the standard Riemannian metric. Define a map $\phi$ of $S^{n}(1)$ into itself by

$$
\phi(p):= \begin{cases}\exp _{p_{0}}(f(\|X\|) X) & \left(p \neq q_{0}\right) \\ q_{0} & \left(p=q_{0}\right)\end{cases}
$$

for $p \in S^{n}(1)$, where $\exp _{p_{0}}$ is the exponential map of $S^{n}(1)$ at $p_{0},\|\cdot\|$ is the norm of - with respect to $g_{1}, X$ is the tangent vector of $S^{n}(1)$ at $p_{0}$ satisfying $\exp _{p_{0}} X=$ $p$ and $\|X\|<\pi$ and $f$ is a $C^{\infty}$-function over $[0, \pi)$ satisfying $1 \leq f \leq 4 / 3$ over $[0, \pi), f^{-1}(4 / 3)=[0, \pi / 4], f^{-1}(1)=[3 \pi / 4, \pi)$ and $f^{\prime}>-1 / \pi$ over $[0, \pi)$. Then, $(f(t) t)^{\prime}=f(t)+f^{\prime}(t) t \geq 1-(t / \pi)>0$ holds over $[0, \pi)$, that is, $f(t) t$ is an increasing function over $[0, \pi)$. Also, we have $\lim _{t \rightarrow \pi-0} f(t) t=\pi$. These facts imply that $\phi$ is a diffeomorphism. Let $M_{\phi}$ be the mapping torus of $\phi$ and $\mathbb{F}$ a foliation on $M_{\phi}$ induced naturally from the foliation $\mathfrak{F}$ on $S^{n}(1) \times[0,1]$ whose leaves are the fibres of the projection of $S^{n}(1) \times[0,1]$ onto $S^{n}(1)$. Denote by $\pi$ the quotient map of $S^{n}(1) \times[0,1]$ onto $M_{\phi}$ and $P$ (resp. $\left.Q\right)$ the projection of $S^{n}(1) \times[0,1]$ onto $S^{n}(1)$ (resp. [0,1]). Also, denote by $g_{2}$ the standard Riemannian metric of $[0,1]$. Define a Riemannian metric $g_{0}$ on $M_{\phi}$ by

$$
g_{0}(X, Y):=u g_{1}\left(P_{*} \tilde{X}, P_{*} \tilde{Y}\right)+(1-u)\left(\phi^{*} g_{1}\right)\left(P_{*} \tilde{X}, P_{*} \tilde{Y}\right)+g_{2}\left(Q_{*} \tilde{X}, Q_{*} \tilde{Y}\right)
$$

for $X, Y \in T_{\pi(p, u)} M_{\phi}$, where $\tilde{X}$ (resp. $\tilde{Y}$ ) is the tangent vector of $S^{n}(1) \times[0,1]$ at $(p, u)$ with $\pi_{*} \tilde{X}=X$ (resp. $\pi_{*} \tilde{Y}=Y$ ) and $\phi^{*} g_{1}$ is the Riemannian metric induced from $g_{1}$ by $\phi$. It is clear that $g_{0}$ is well-defined. Take an arbitrary vertical curve $\alpha$ in $M_{\phi}$. Since $g_{1}\left(\phi_{*} X ; \phi_{*} X\right) \leq(4 / 3)^{2} g_{1}(X, X)$ for every $X \in T S^{n}(1)$, we see that $G_{\alpha}^{\perp}(\delta) \leq(4 / 3)^{[l(\alpha)]+1}$ for every $\delta \in \operatorname{Rec}(\alpha, \cdot)$, that is, $\sup G_{\alpha}^{\perp} \leq(4 / 3)^{[l(\alpha)]+1}<$ $\infty$, where $l(\alpha)$ is the length of $\alpha$ with respect to $g_{0}$ and [.] is the Gauss's symbol of . (see Figure 3.7). 


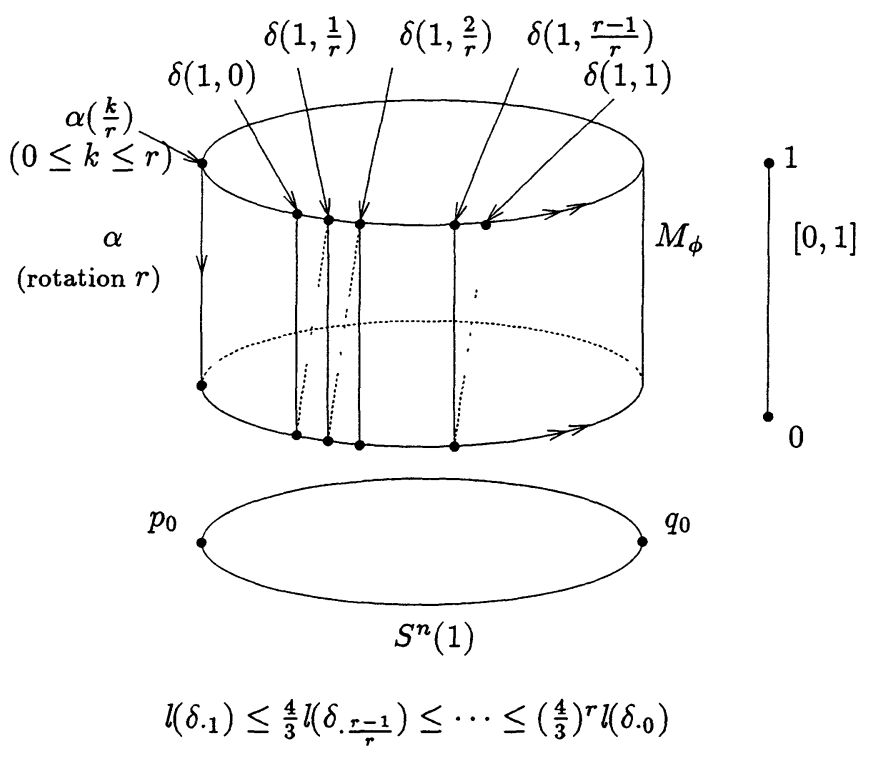

Figure 3.7.

Thus $\mathfrak{F}$ satisfies the assumption of Theorem 2 with respect to $g_{0}$.

Take an arbitrary Riemannian metric $g$ on $M_{\phi}$. Let $\alpha_{0}$ be a vertical curve defined by $\alpha_{0}(s)=\pi\left(p_{0}, 1-s\right)$ and $\beta_{0}$ be a horizontal curve (with respect to $g)$ satisfying $\beta_{0}(0)=\alpha_{0}(0), \quad \beta_{0}(1) \in \pi\left(P^{-1}\left(\exp _{p_{0}}(\pi / 4) X_{0}\right)\right)$ and $\beta_{0}([0,1]) \subset$

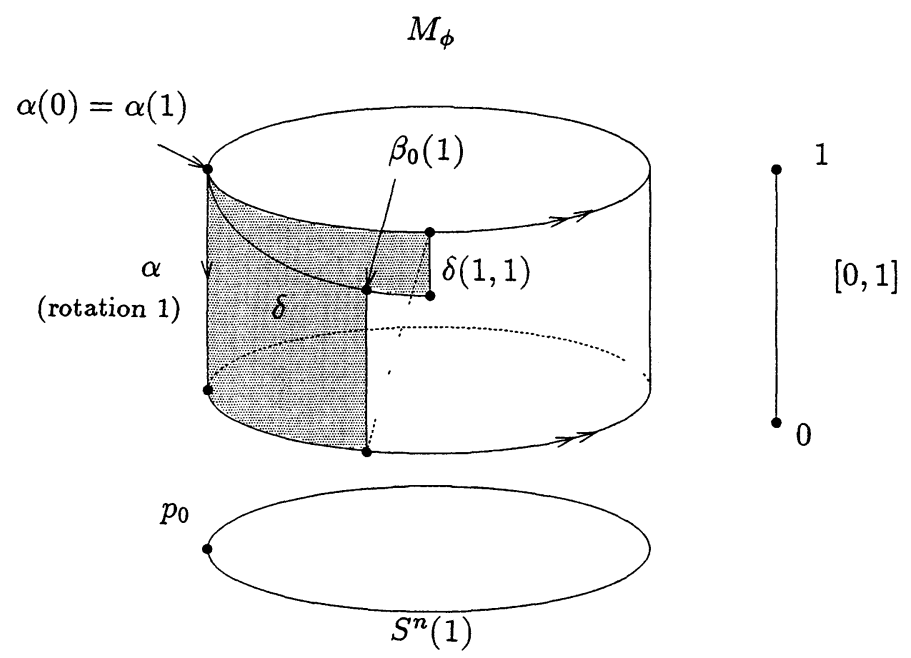

Figure 3.8. 
$\pi\left(P^{-1}\left(\left\{\exp _{p_{0}}\left((\pi t / 4) X_{0}\right) \mid t \in[0,1]\right\}\right)\right)$, where $X_{0}$ is some unit tangent vector of $S^{n}(1)$ at $p_{0}$. Let $\delta$ be the rectangle with $\delta_{0}=\alpha_{0}$ and $\delta_{.0}=\beta_{0}$. Then we have $G_{\alpha_{0}}^{\perp}(\delta)>1$ (see Figure 3.8). Thus any Riemannian metric $g$ on $M_{\phi}$ is not bundle-like for $\mathfrak{F}$, that is, $\mathfrak{F}$ is not a Riemannian foliation.

Similarly, we can give examples showing the topological gap between totally geodesicable foliations and foliations admitting a Riemannian metric satisfying the condition $(\mathrm{V})$ in Introduction.

\section{REFERENCES}

[ 1 ] R. A. Blumenthal and J. J. HebDa, De Rham decomposition theorems for foliated manifolds, Ann. Inst. Fourner (Grenoble), 33 (1983), 183-198.

[2] R. A. Blumenthal and J. J. Hebda, Ehresmann connection for foliations, Indiana Univ. Math. J., 33 (1984), 597-612.

[3] R. A. Blumenthal and J. J. Hebda, Complementary distributions which preserve the leaf geometry and applications to totally geodesic folations, Quart. J. Math. Oxford Ser. (2), 35 (1984), 383-392.

[4] R. A. Blumenthal and J. J. HebDa, An analogue of the holonomy bundle for a foliated manifold, Tôhoku Math. J., 40 (1988), 189-197

[5] R. Botт, Lectures on characteristic classes and foliations, Lecture Notes in Math, 279, Springer-Verlag, 1972, 1-80.

[6] G. CaIRNs, Totally umbilic Riemannian foliatıons, Michıgan Math. J., 37 (1990), 145-159.

[7] C. Ehresmann, Les connexions infinitésımales dans un éspace fibré différentiable, Colloque de Topologie, Bruxelles, 1950, Georges Thone, Liege; Masson et Cie., Parıs, 1951, 29-55.

[8] R. HeRmanN, On the differential geometry of foliations, Ann. of Math., 72 (1960), 445-457

[9] D. L. Johnson AND L. B. Whitt, Totally geodesic foliations, J. Differential Geom., 15 (1980), 225-235.

[10] N. KoIKE, Totally umbilic foliations and decomposition theorems, Sattama Math. J., 8 (1990), 1-18.

[11] N. KoIKE, Foliations on a Riemannian manifold and Ehresmann connections, Indiana Univ. Math. J., 40 (1991), 277-292.

[12] N. KoIKE, Totally umbilic orthogonal nets and decomposition theorems, Sattama Math. J., 10 (1992), 1-19.

[13] N. KoIKE, Ehresmann connections for a foliation on a manifold with boundary, SUT J. Math., 30 (1994), 147-158.

[14] P Molino, Riemannian Foliatıons, Progress in Math., 73, Birkhäuser, 1988.

[15] B. Reinhart, Foliated manifolds with bundle-like metrics, Ann. of Math., 69 (1959), 119 132.

[16] V RovenskiI, Foliations on Riemannian Manifolds and Submanifolds, Birkhäuser, Boston, 1998.

DEPARTMENT OF MATHEMATICS

FACULTY OF SCIENCE

SCIENCE UNIVERSITY OF TOKYO

26 WaKamiYa ShinJukU-KU

TOKYO 162-0827 JAPAN 